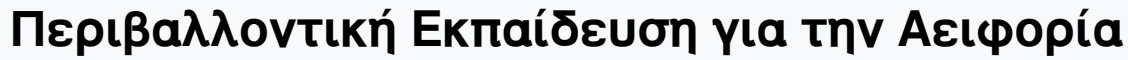

Tóp. 1, Ap. 1 (2019)

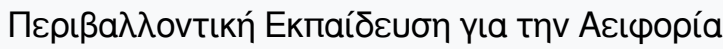

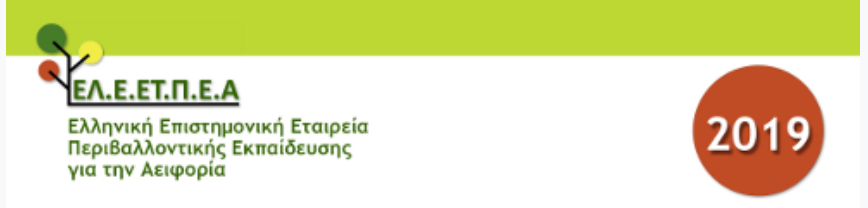

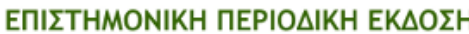

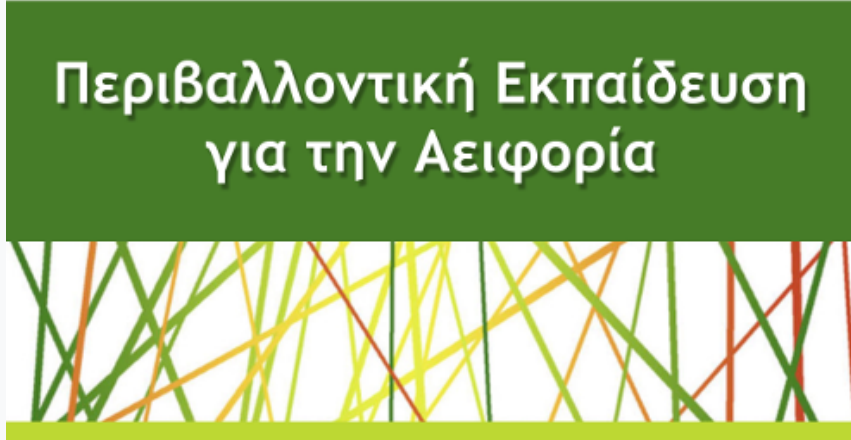

ISSN: $2585-3813$

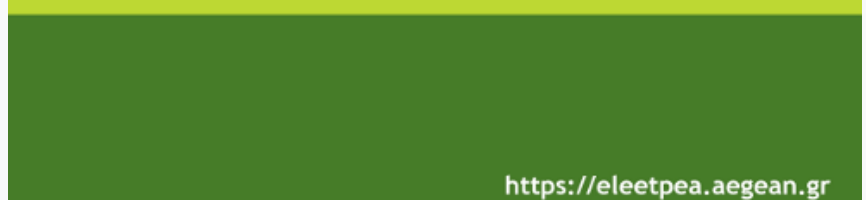

https://ejournals.epublishing.ekt.gr/index.php/enveducation

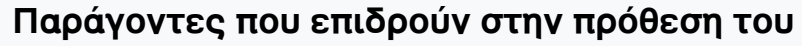

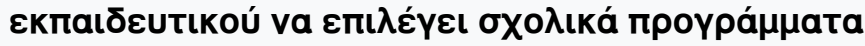

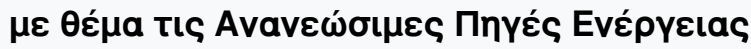

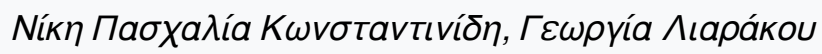
doi: $10.12681 /$ ees.16846

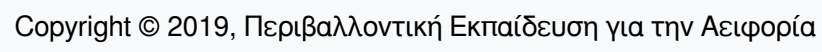

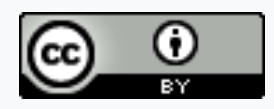

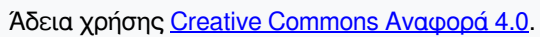

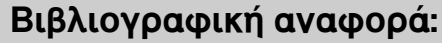

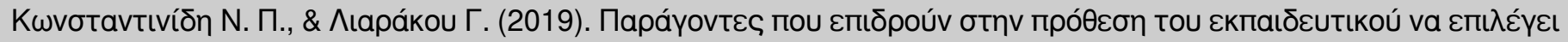

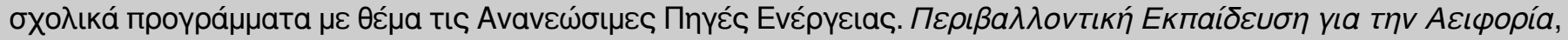
1(1), 1-13. https://doi.org/10.12681/ees.16846 


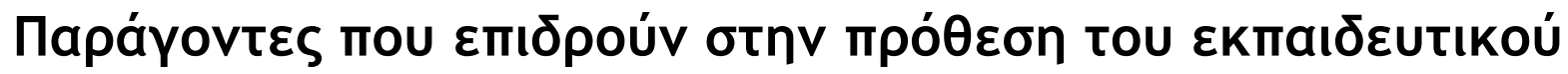

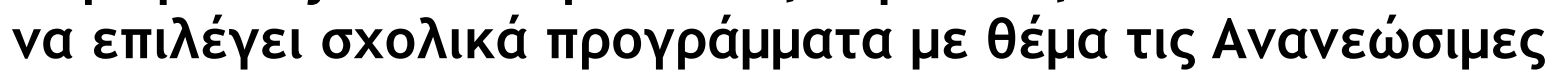

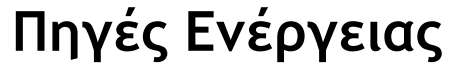

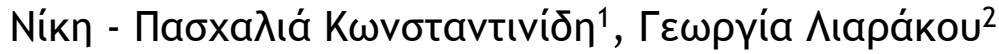

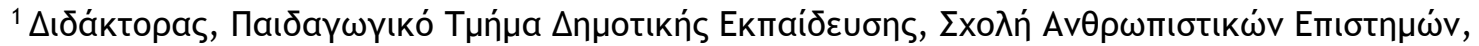

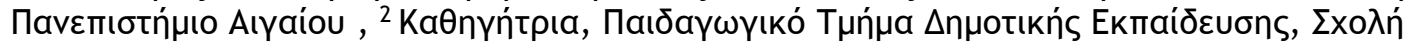

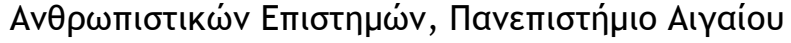

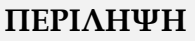

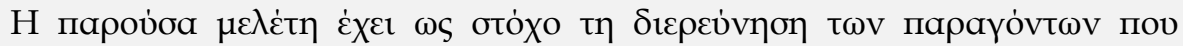

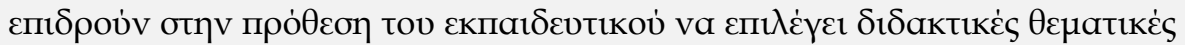

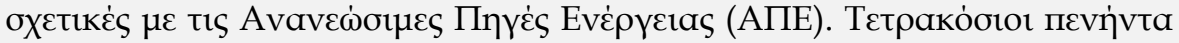

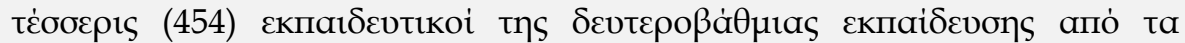

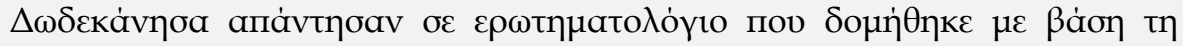

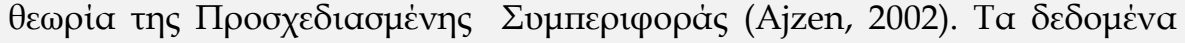

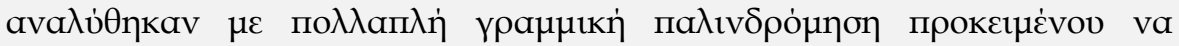

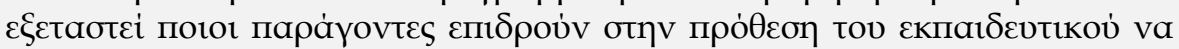

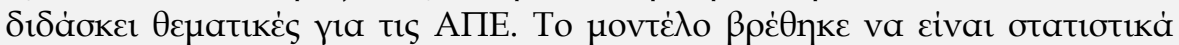

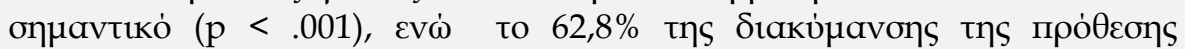

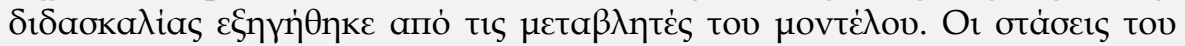

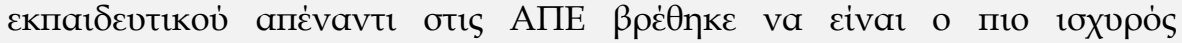

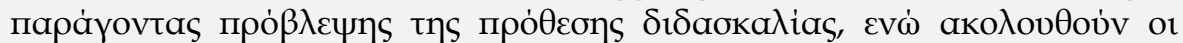
avtı

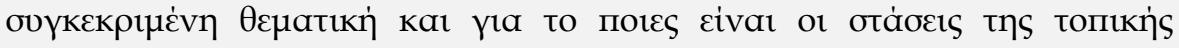

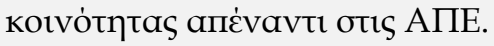

\author{
$\Lambda$ EEEI $\Sigma$-K $\Lambda$ EI $\Delta \mathrm{IA}:$

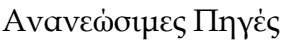 \\ Evépүદıаs,

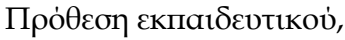

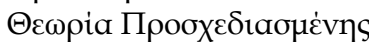 \\ इ
}

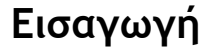

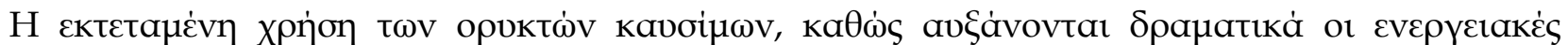

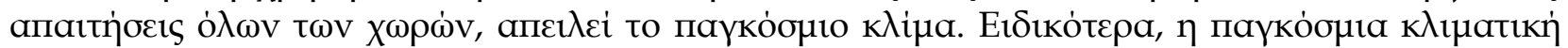

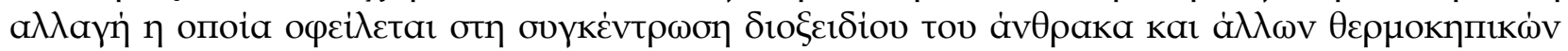

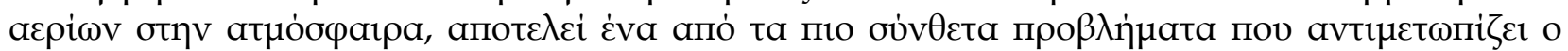

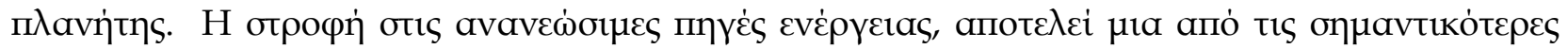

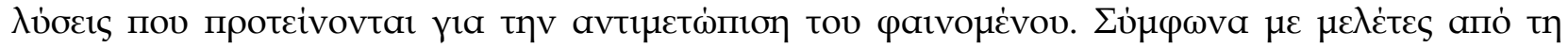

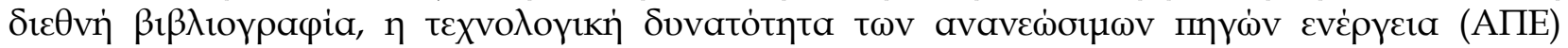

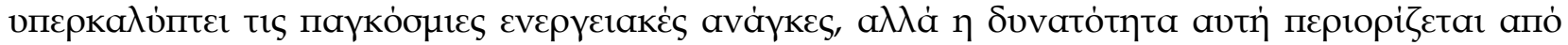

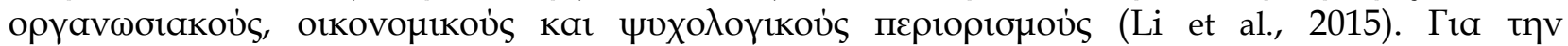

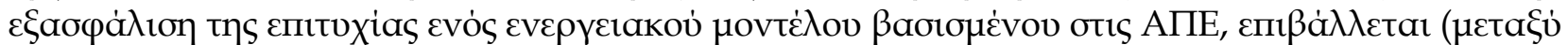

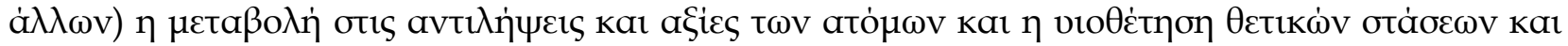

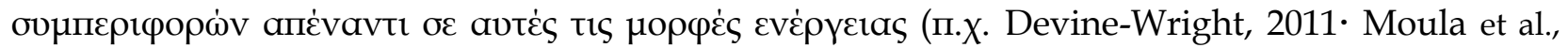
2013).

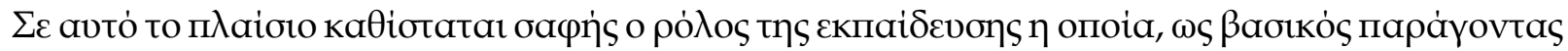

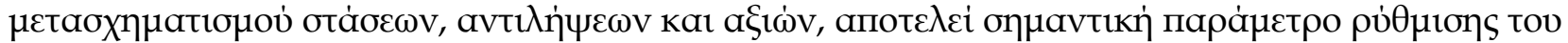

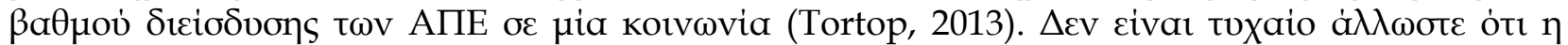
UNESCO (2005)

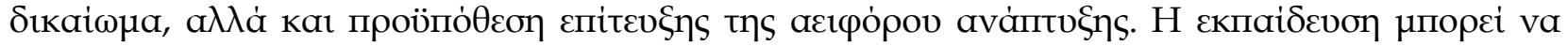




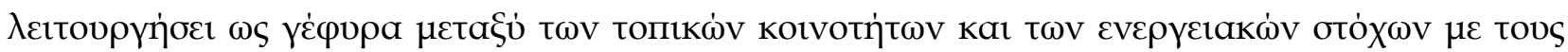

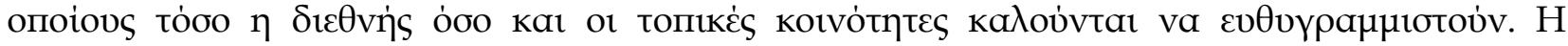

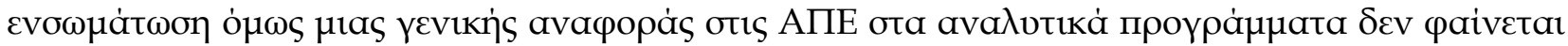

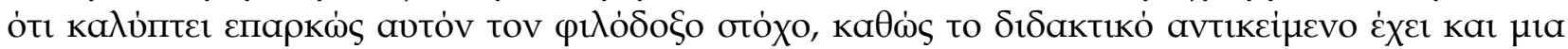

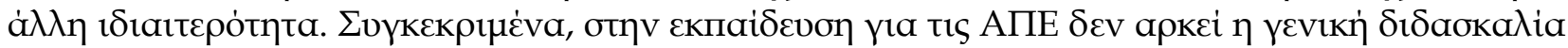

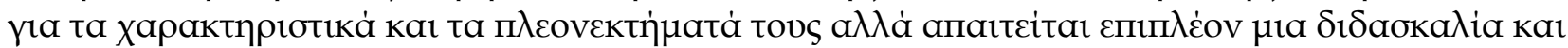

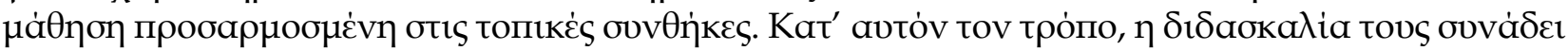

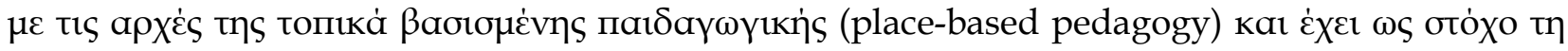

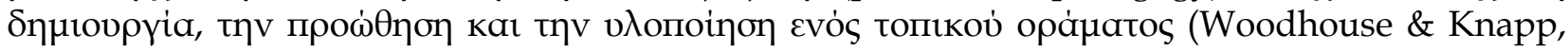
2013. Gruenewald, 2016).

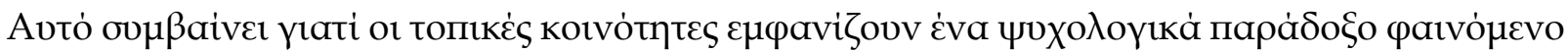

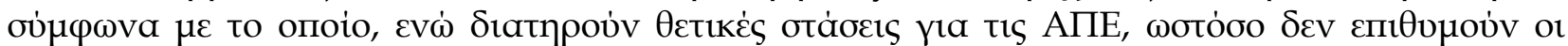

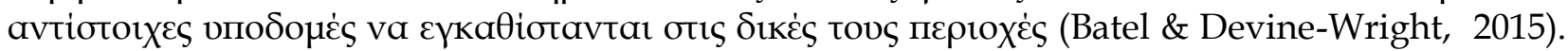

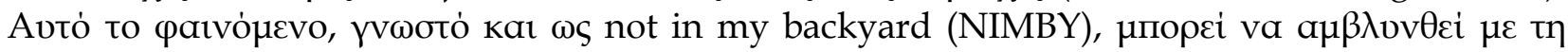

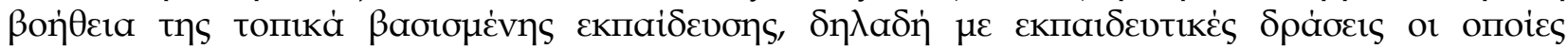

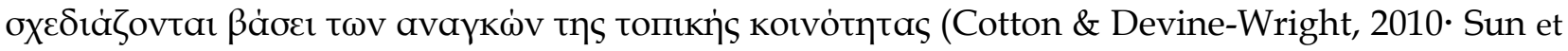
al., 2016).

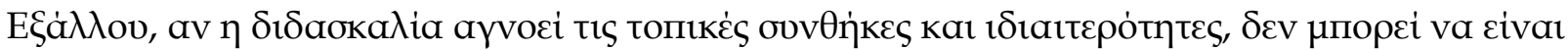

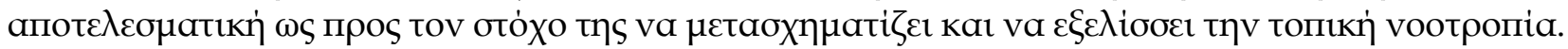

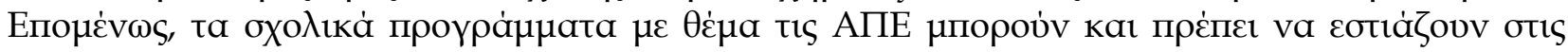

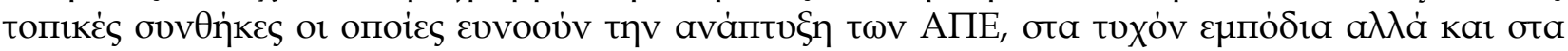

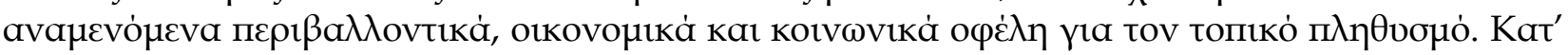

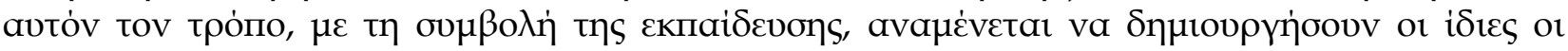

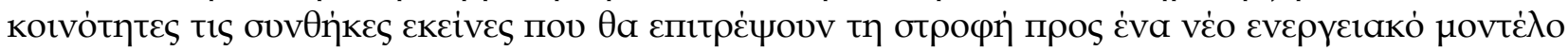

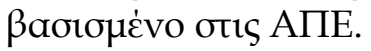

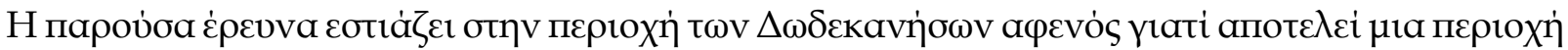

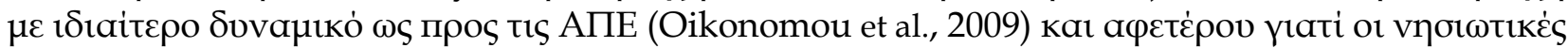

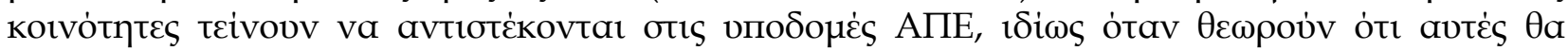

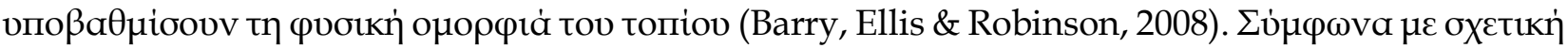

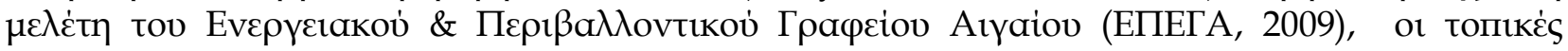

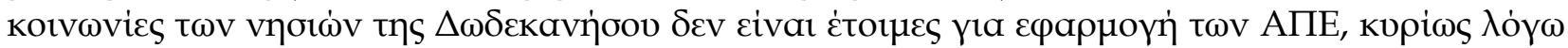

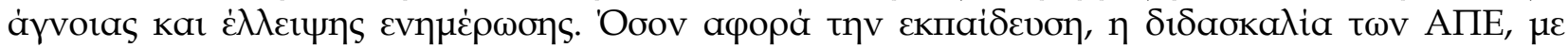

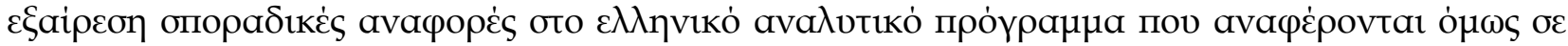

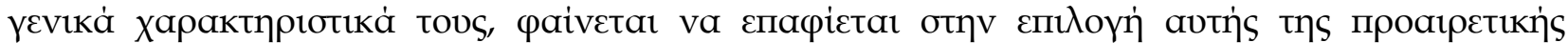

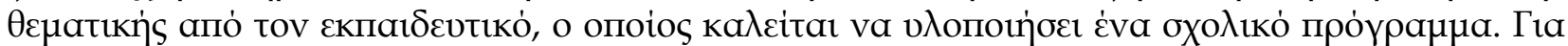

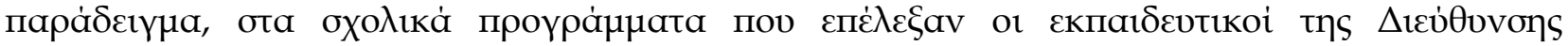

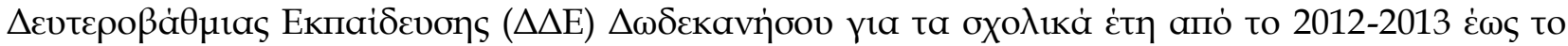

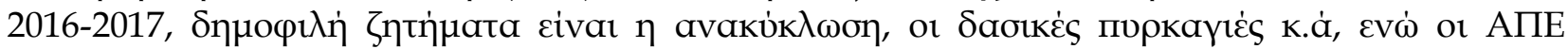

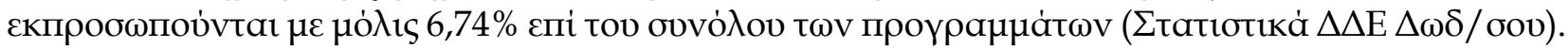

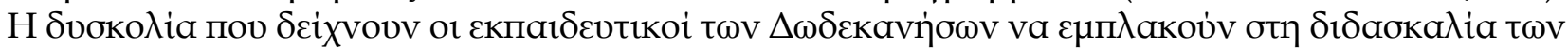

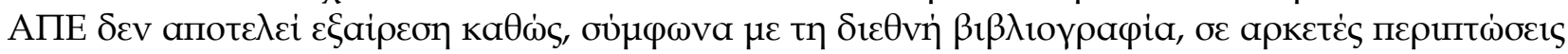

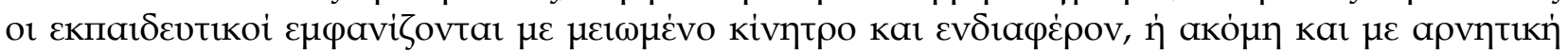

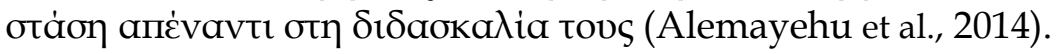

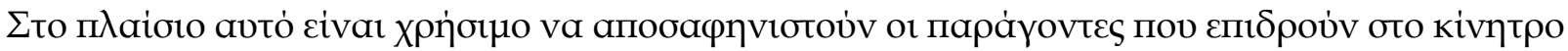

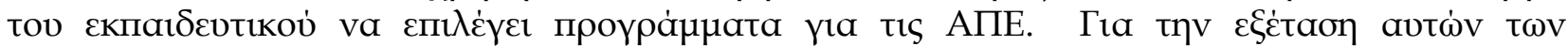

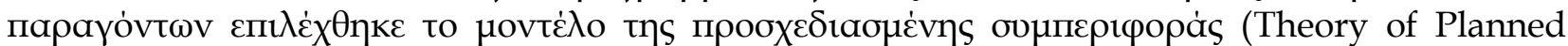

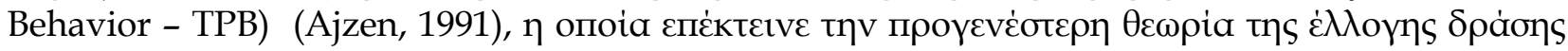

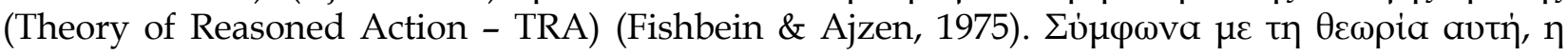

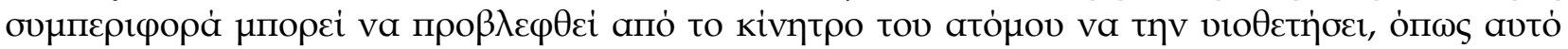

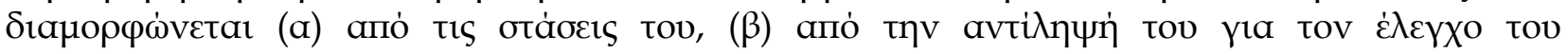

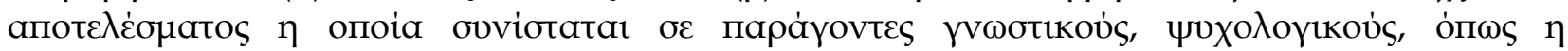

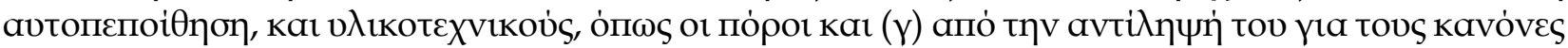




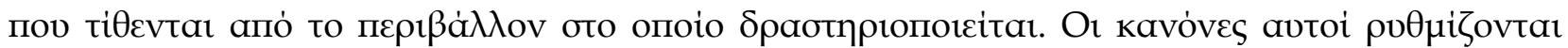

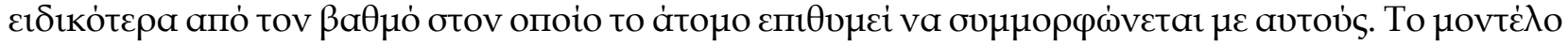

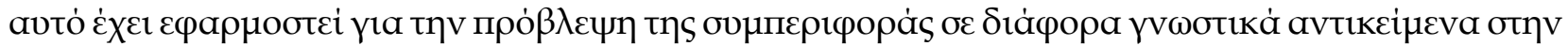

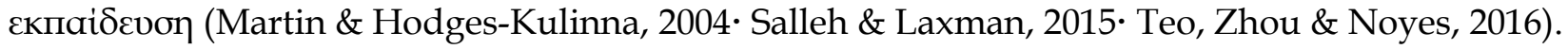

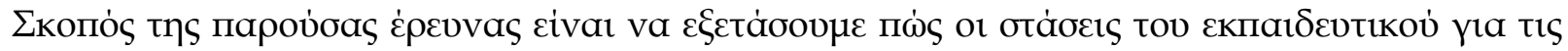

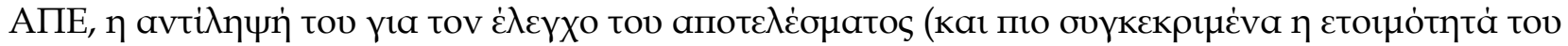

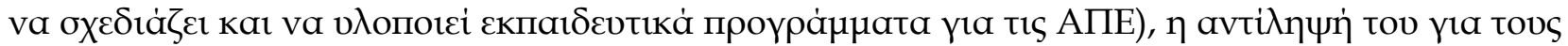

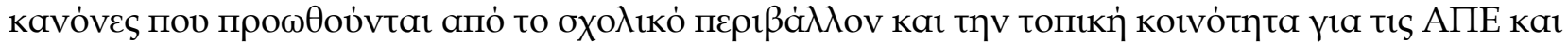

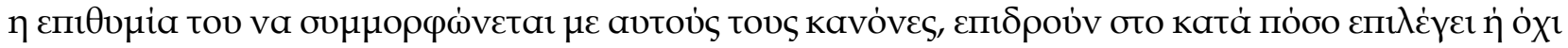

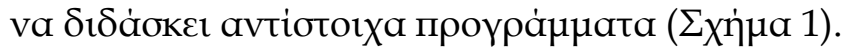

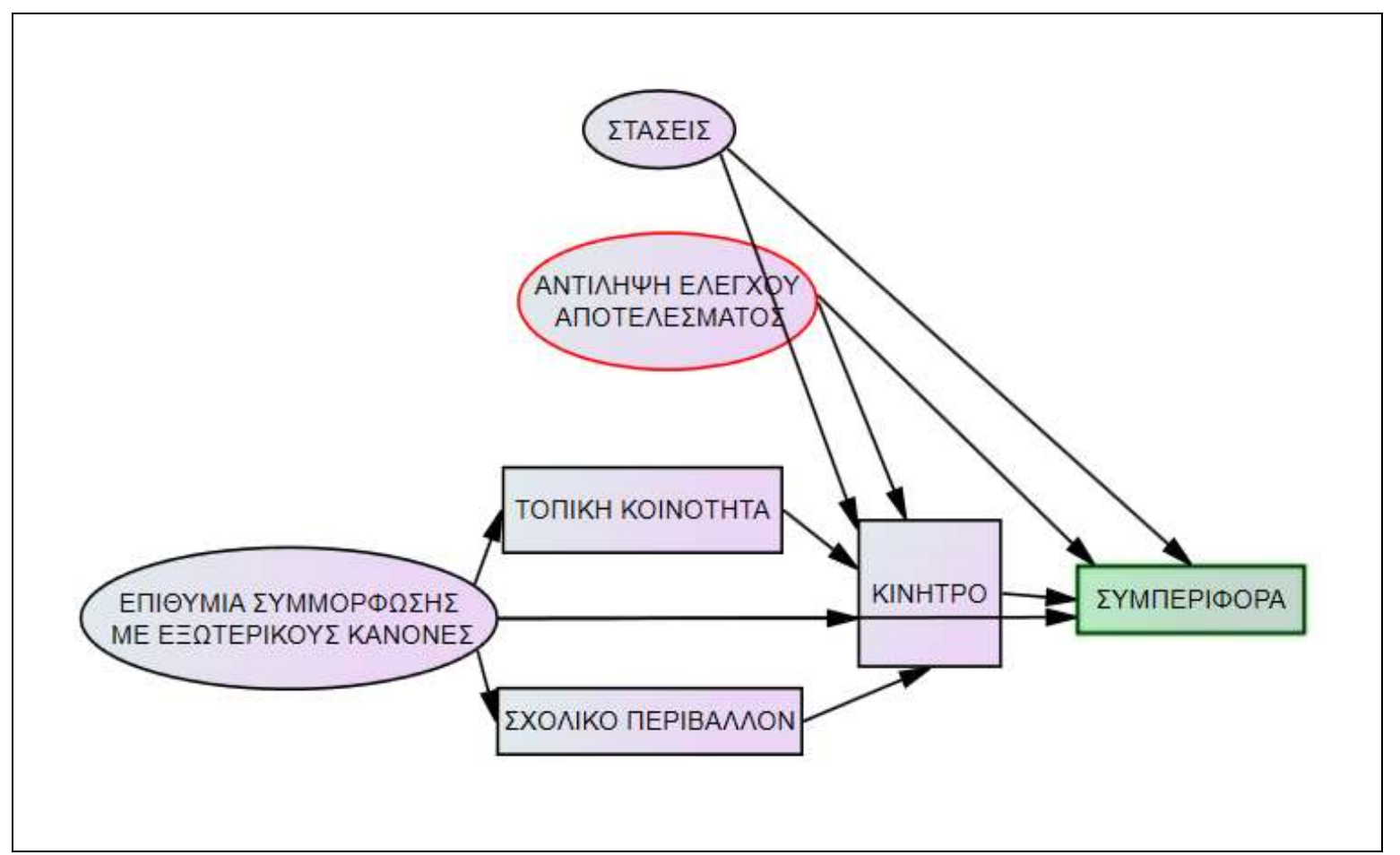

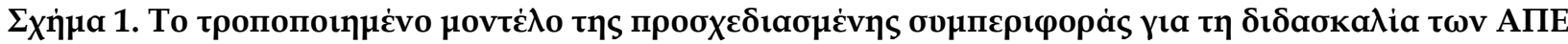

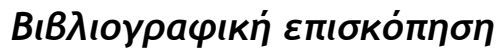

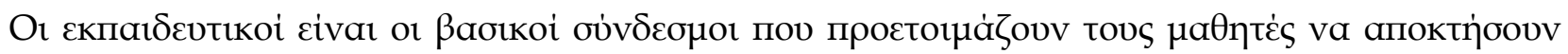

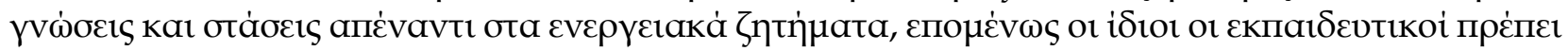

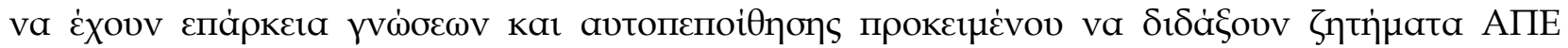

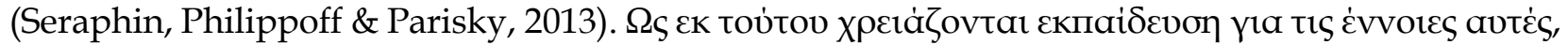

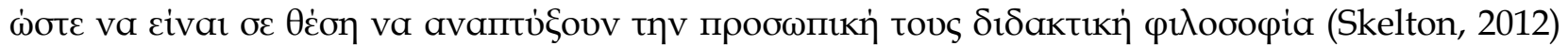

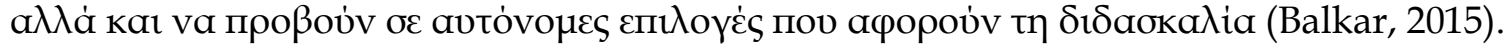

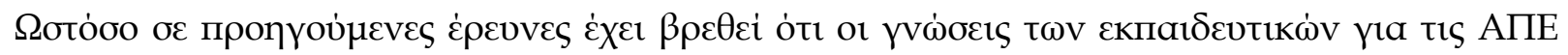

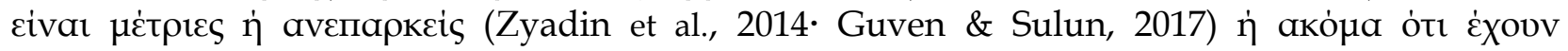

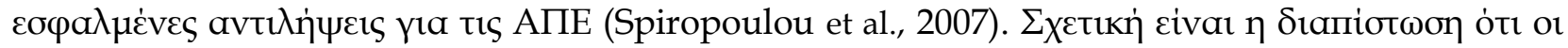

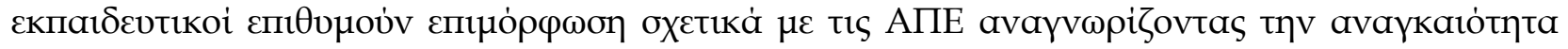

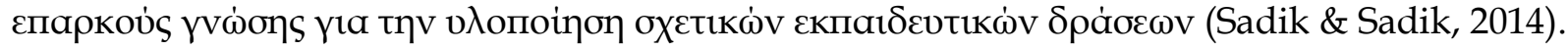

'Oøov a

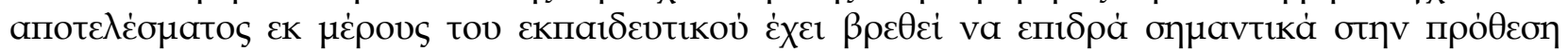

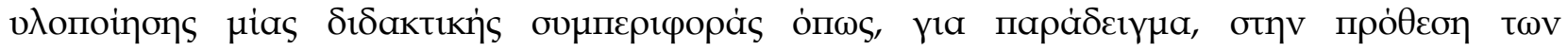

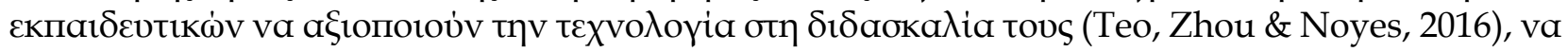

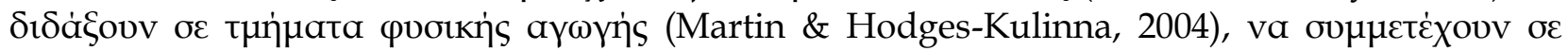




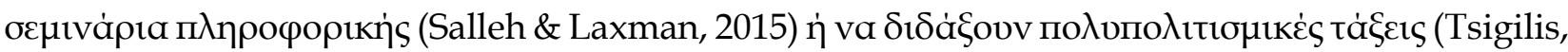
Tsioumis \& Gregoriadis, 2006).

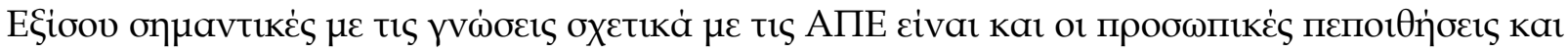

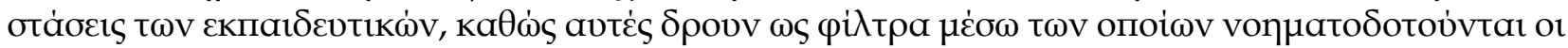

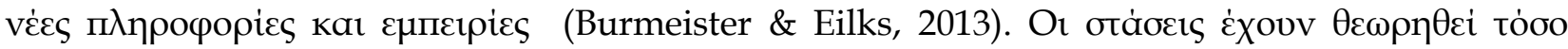

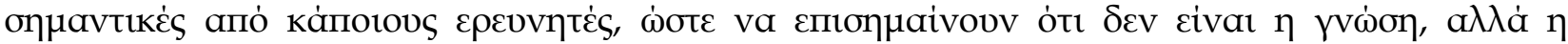

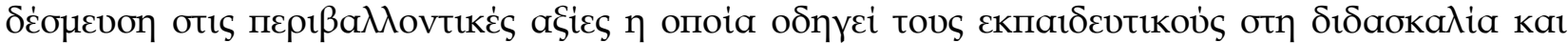

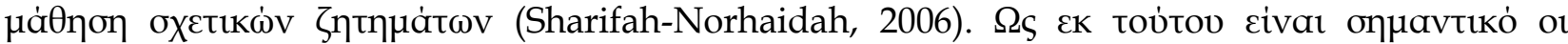

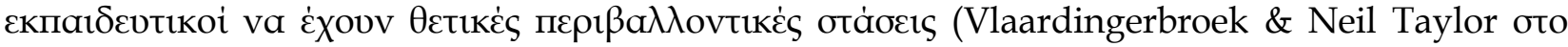

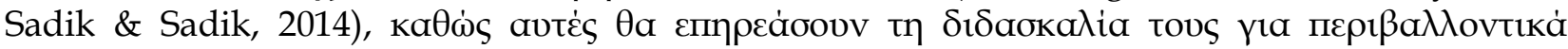

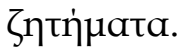

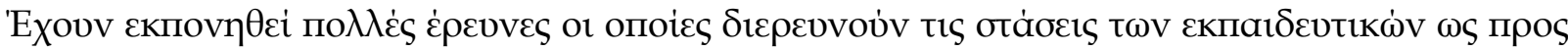

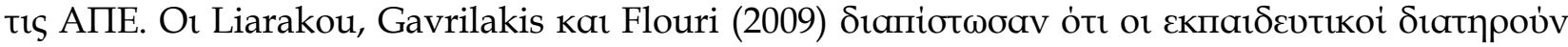

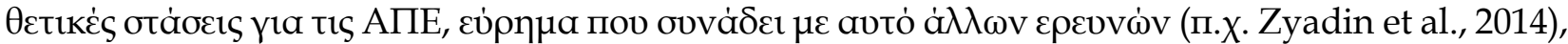

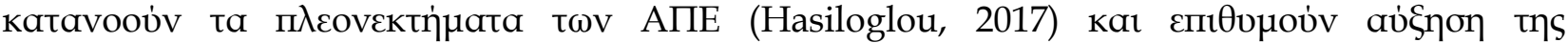

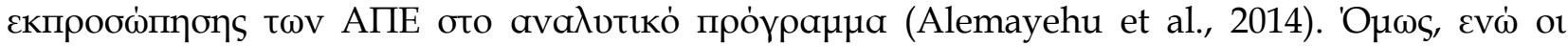

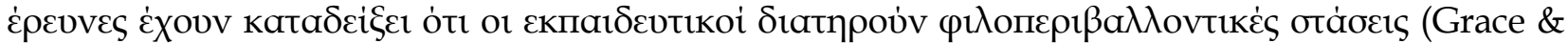

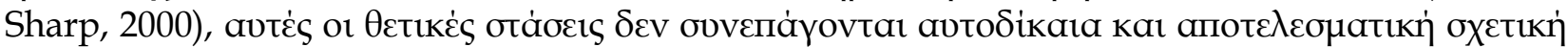

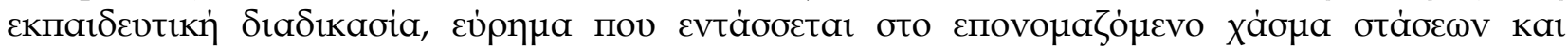

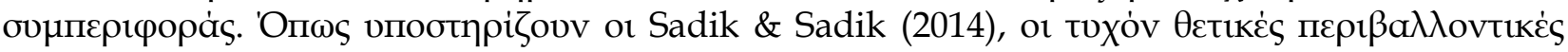

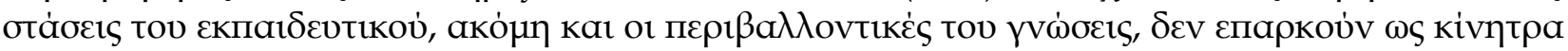

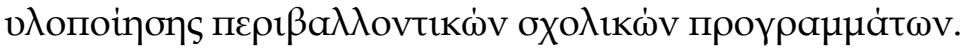

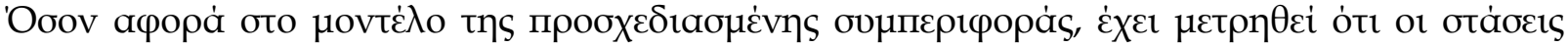

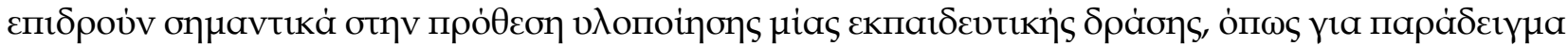

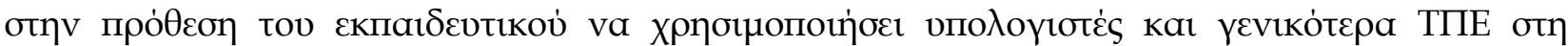

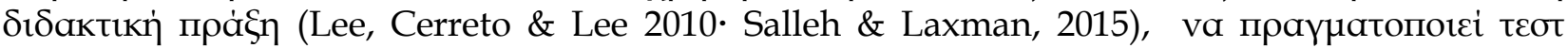

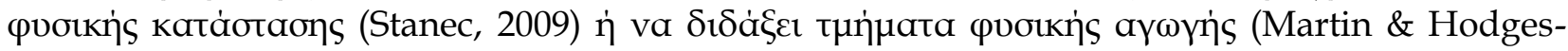
Kulinna, 2004).

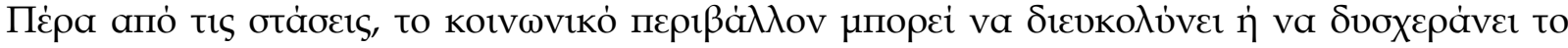

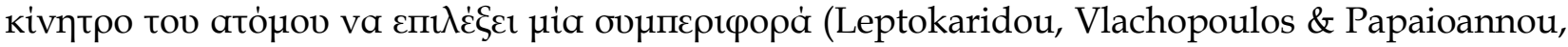

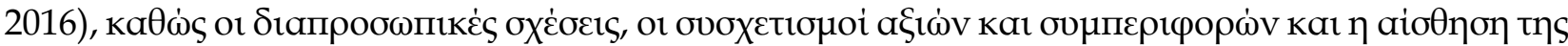

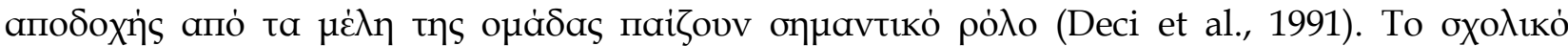

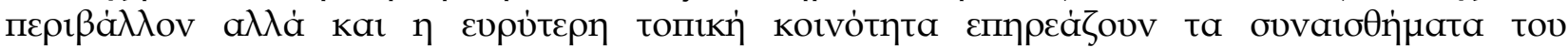

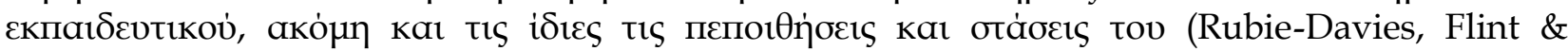

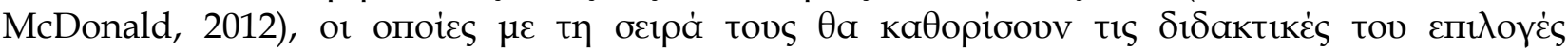

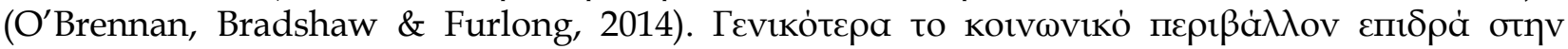

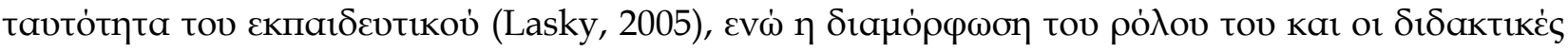

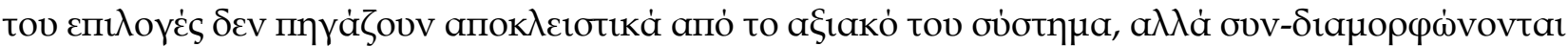

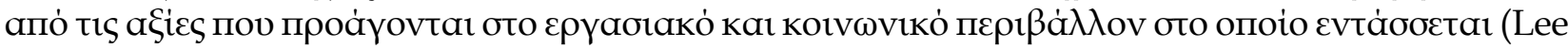
\& Nie, 2017).

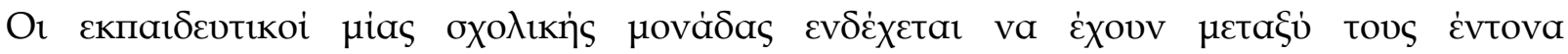

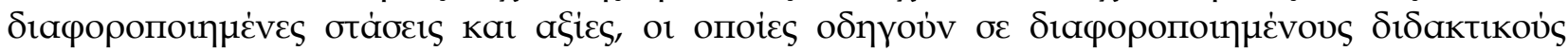

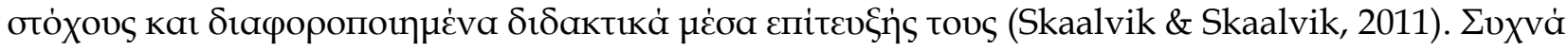

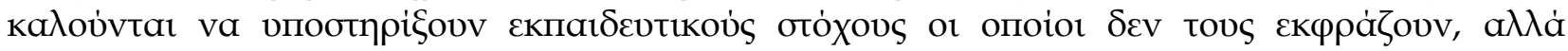

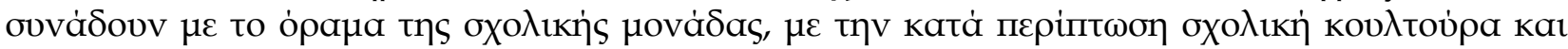

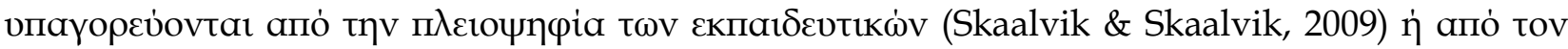

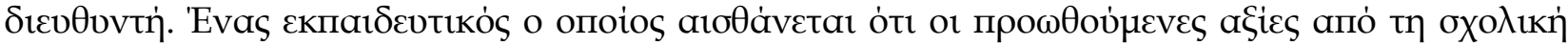

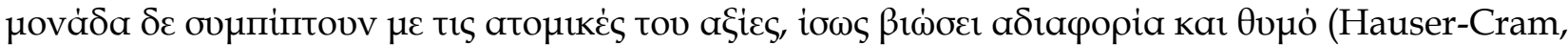

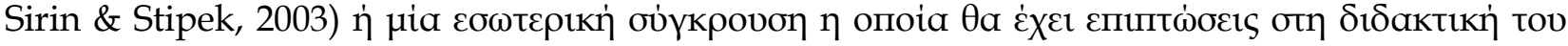
єпiठoon (Skaalvik \& Skaalvik, 2011). 


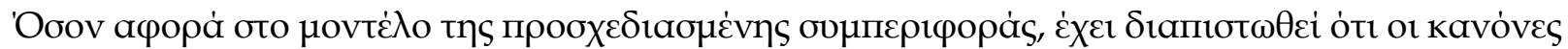

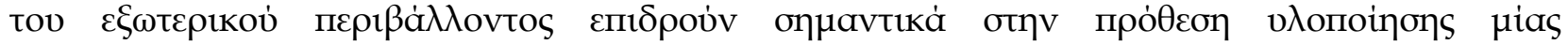

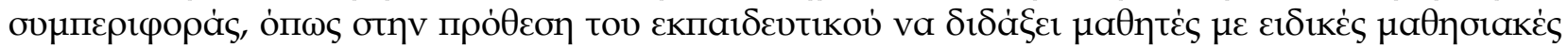

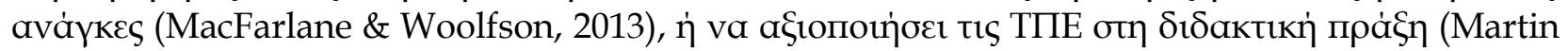
\& Hodges-Kulinna, 2004• Salleh \& Albion, 2004· Salleh \& Laxman, 2015).

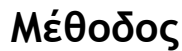

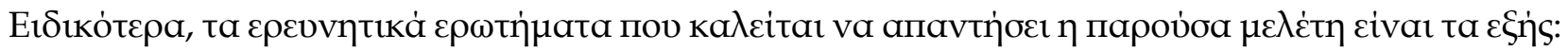

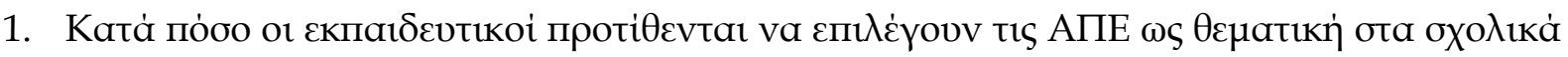

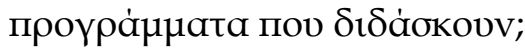

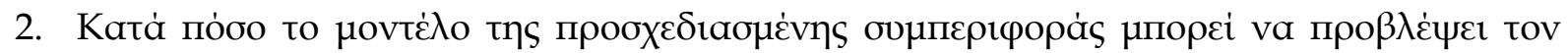

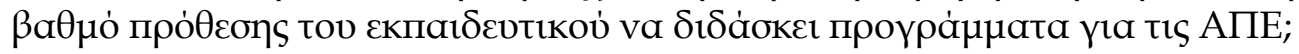

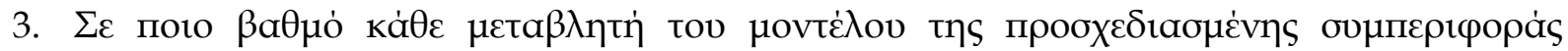

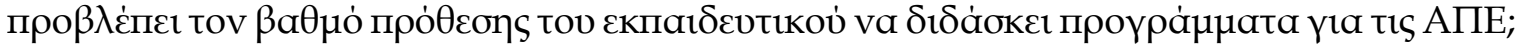

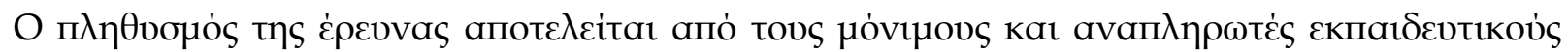

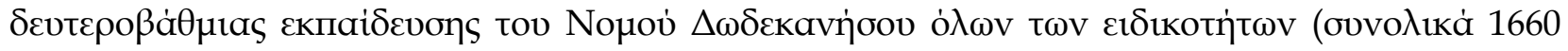

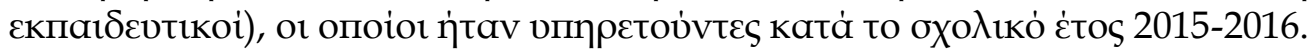

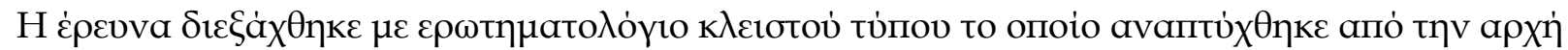

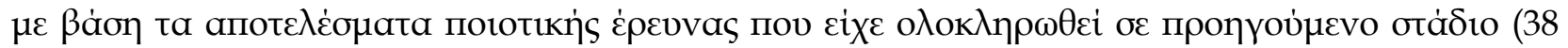

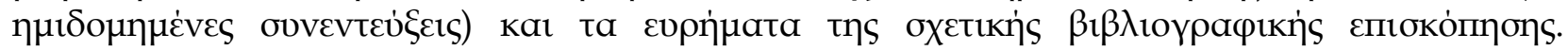

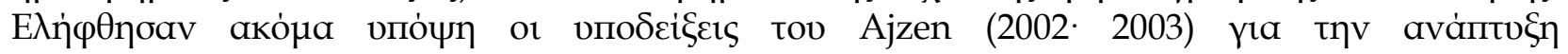

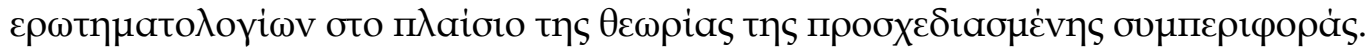

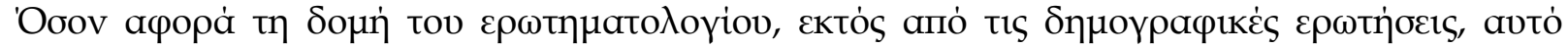

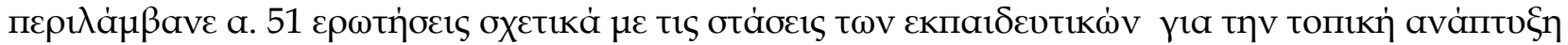

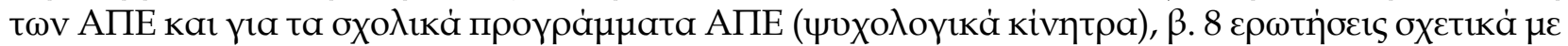

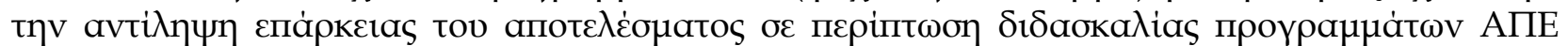

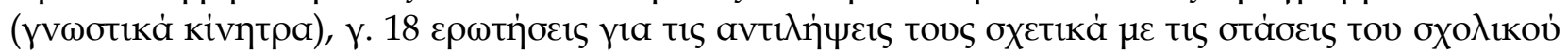

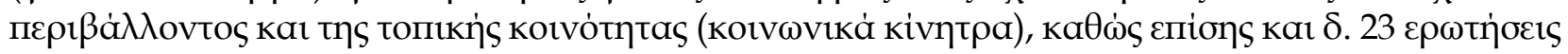

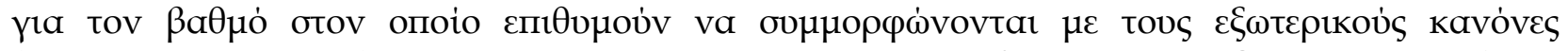

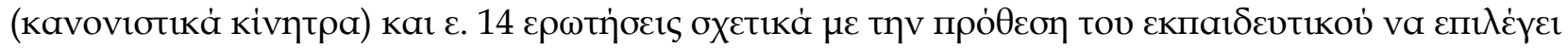

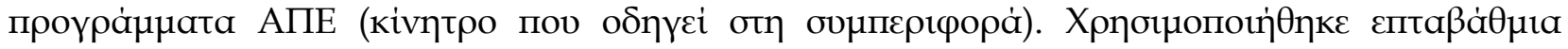

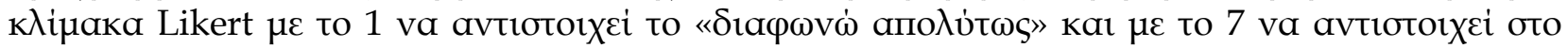

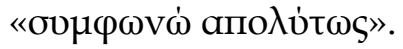

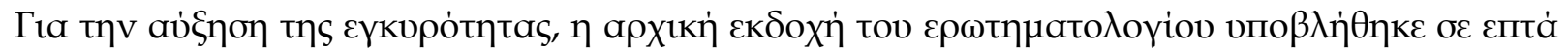

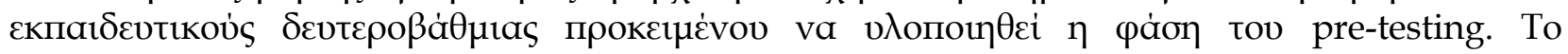

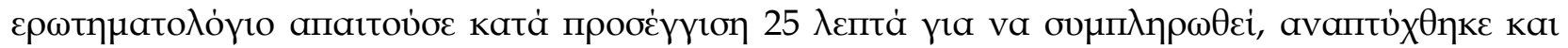

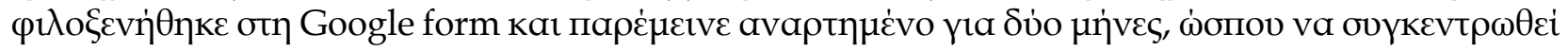

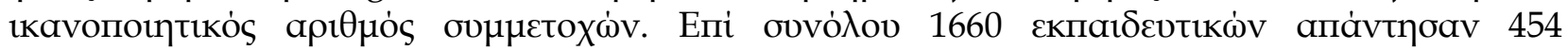

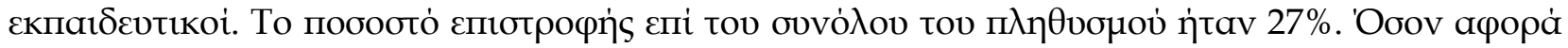

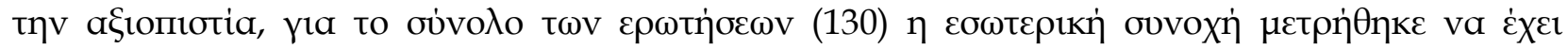

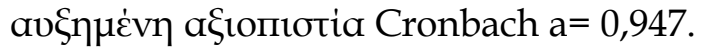

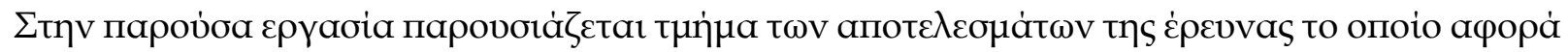

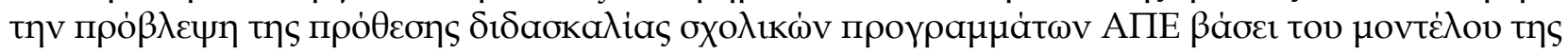

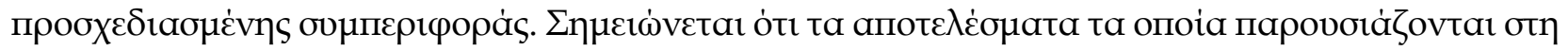

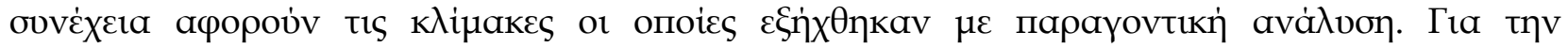

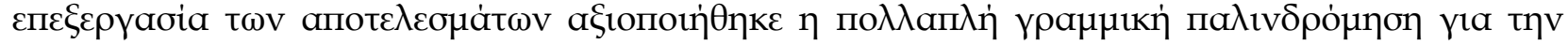

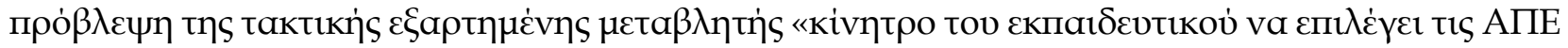

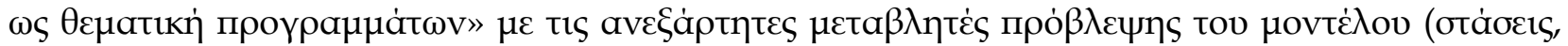

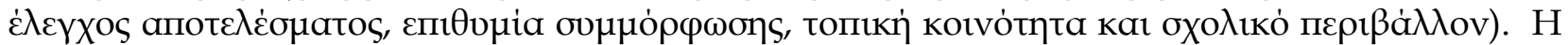

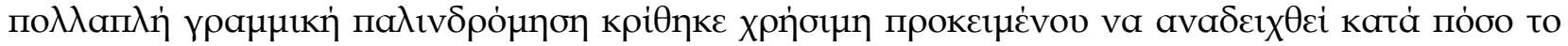




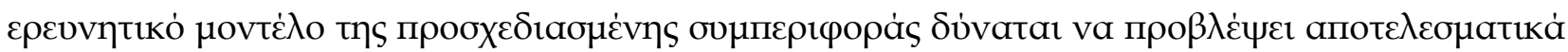

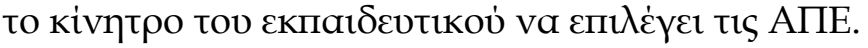

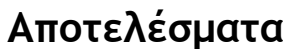

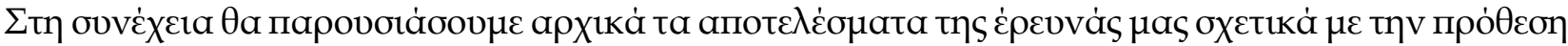

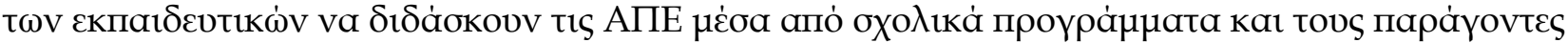

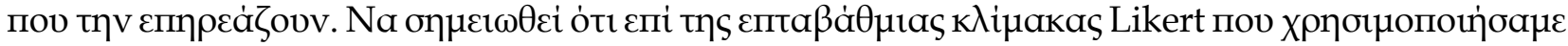

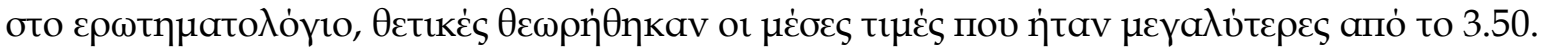

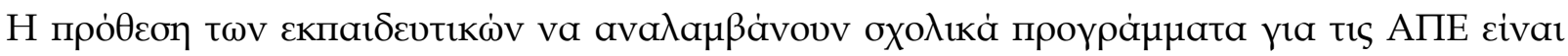

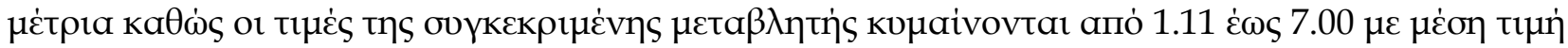

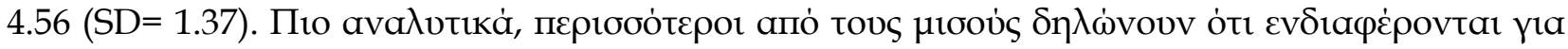

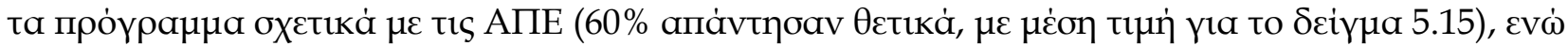

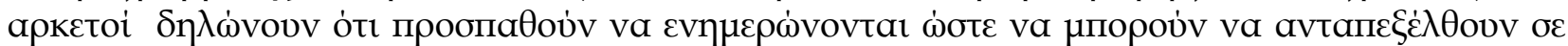

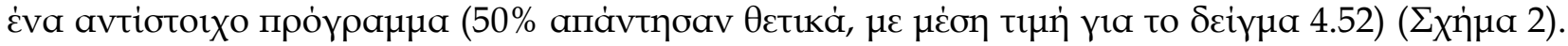

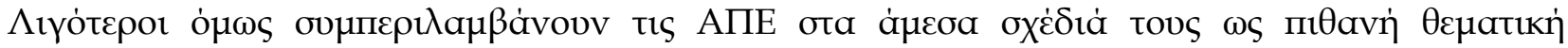

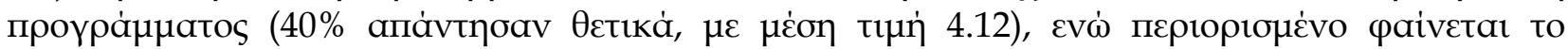

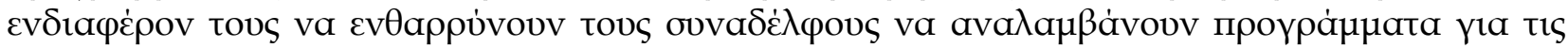

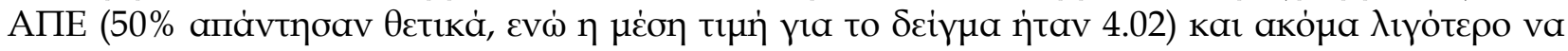

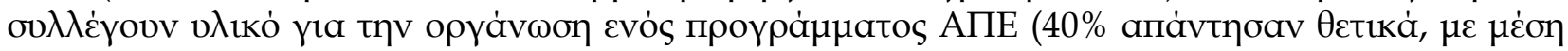

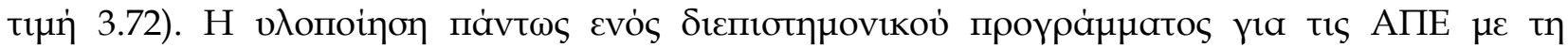

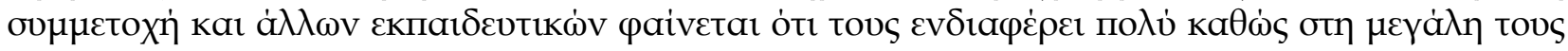

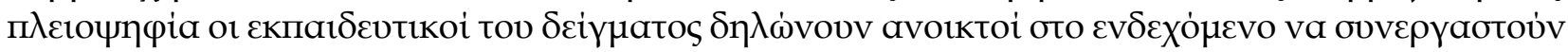

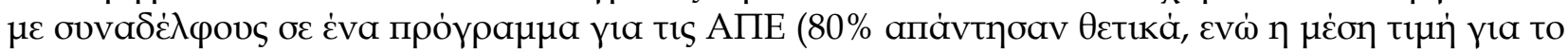

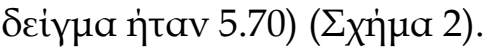

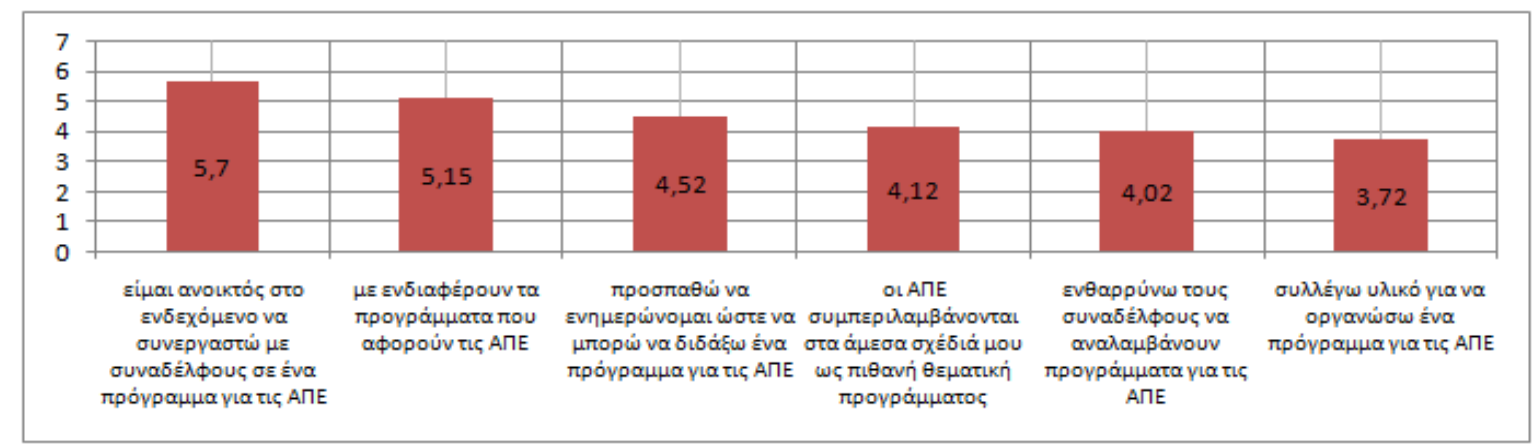

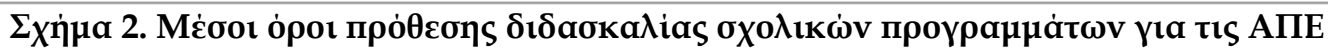

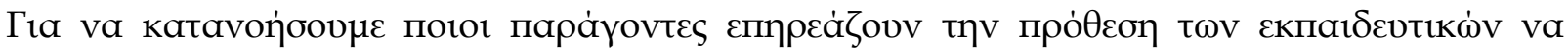

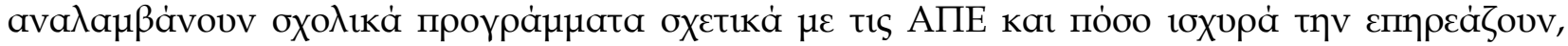

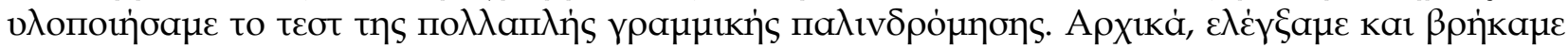

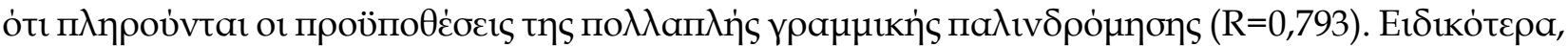

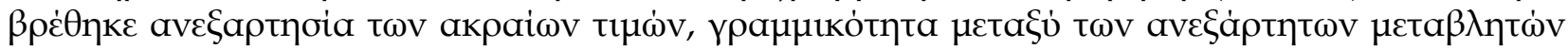

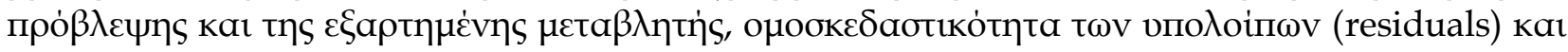

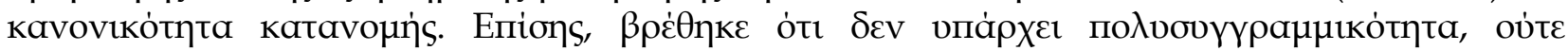

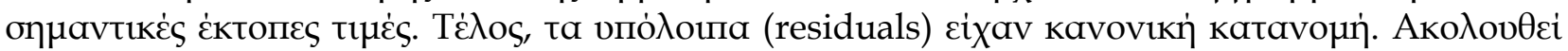

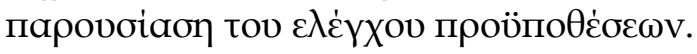




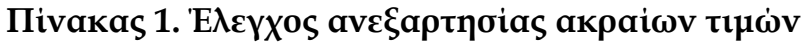

\begin{tabular}{llcccccc}
\hline $\mathbf{R}$ & $\mathbf{R}^{2}$ & $\begin{array}{c}\text { Adjust } \\
\text { ed R }\end{array}$ & $\begin{array}{c}\text { Std. Error } \\
\text { of the }\end{array}$ & & \multicolumn{4}{c}{ Change Statistics } \\
\cline { 5 - 7 } & & & Estimate & $\begin{array}{c}\mathrm{R}^{2} \\
\text { Change }\end{array}$ & F Change & df1 & df2 \\
\hline 0,793 & 0,628 & 0,624 & 0,841 & 0,628 & 151,515 & 5 & 448 \\
\hline
\end{tabular}

Sig F change $=0,000$, Durbin-Watson $=2,108$

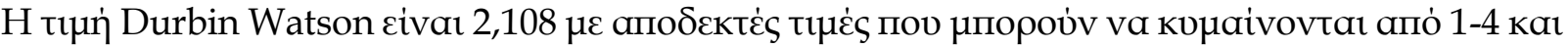

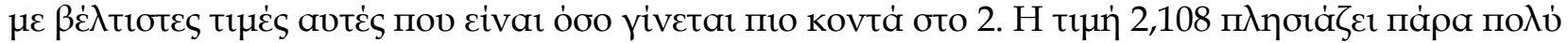

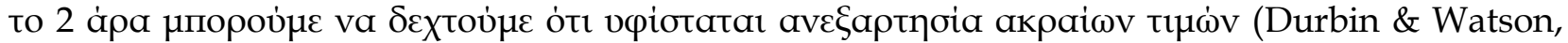
1950) (Пivakas 1 ).

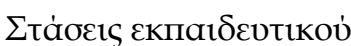

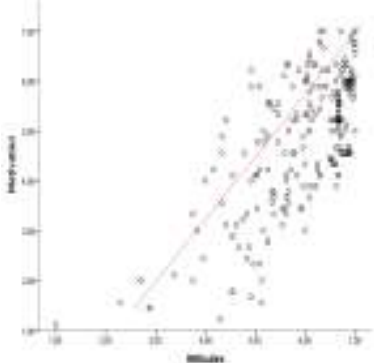

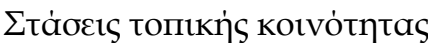

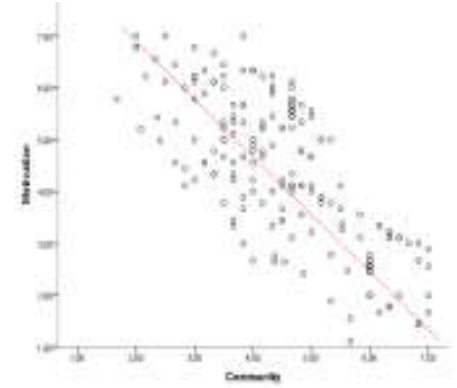

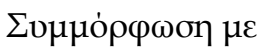

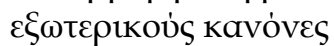

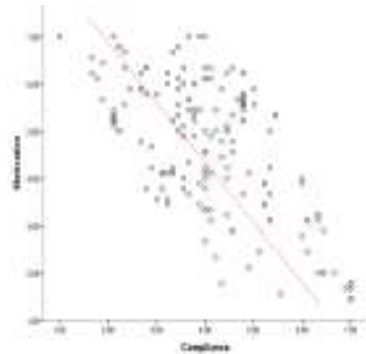

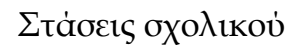

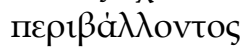

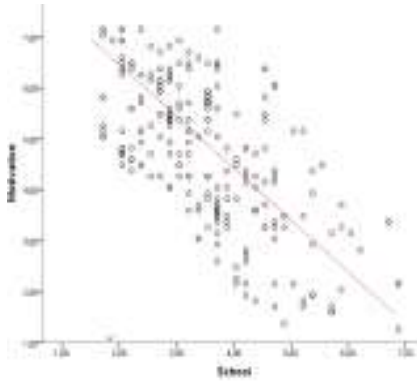

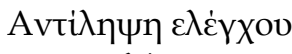

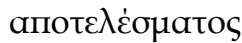

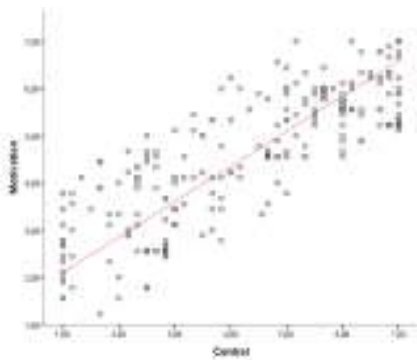

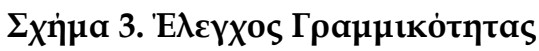

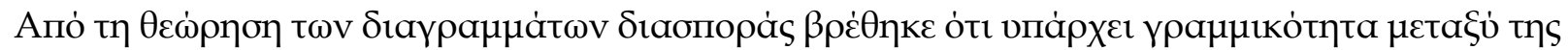

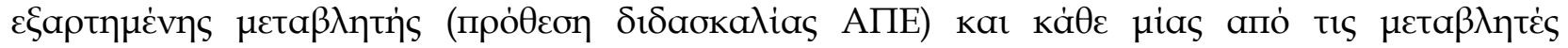

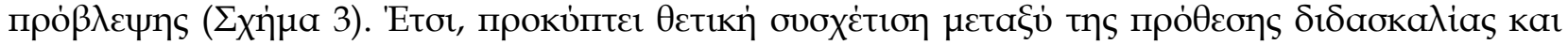

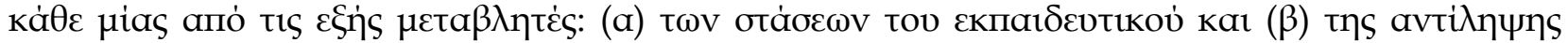

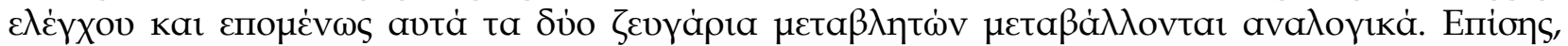

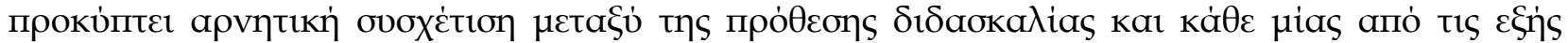

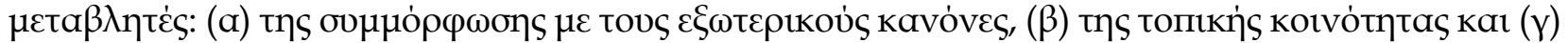

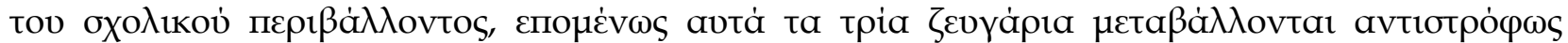

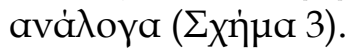




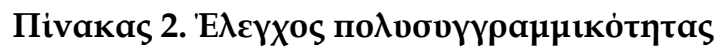

\begin{tabular}{|c|c|c|}
\hline 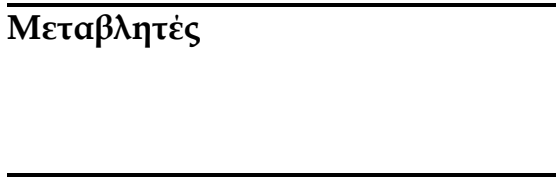 & Avo $\chi \dot{\eta}$ (Tolerance) & 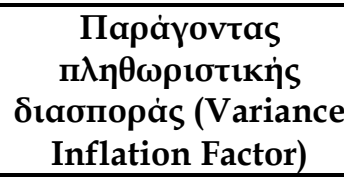 \\
\hline 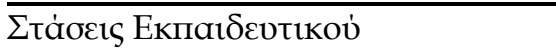 & 0,857 & 1,166 \\
\hline 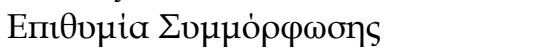 & 0,764 & 1,309 \\
\hline 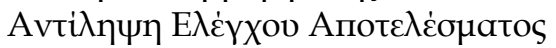 & 0,771 & 1,297 \\
\hline 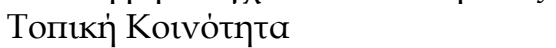 & 0,715 & 1,399 \\
\hline 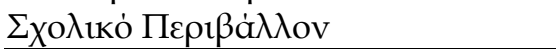 & 0,837 & 1,194 \\
\hline
\end{tabular}

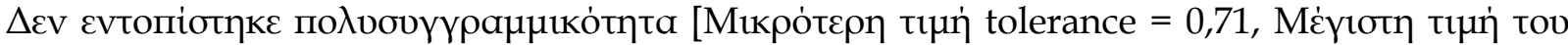

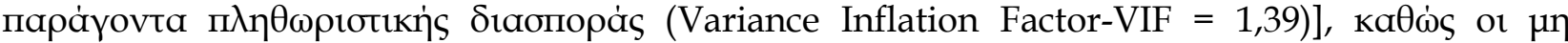

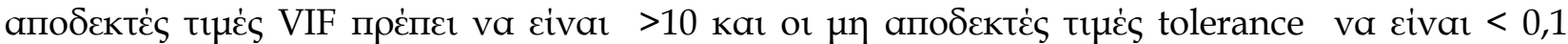
(Пivakas 2).

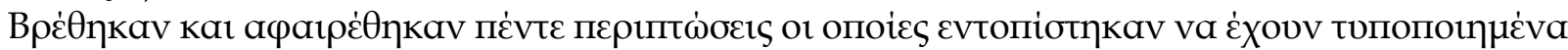

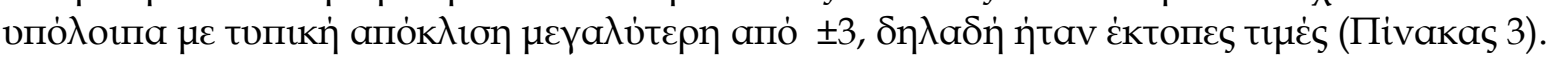

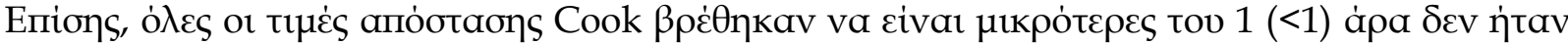

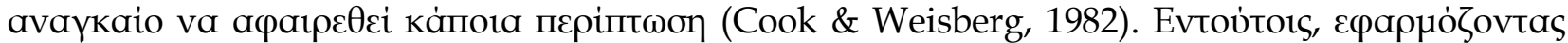

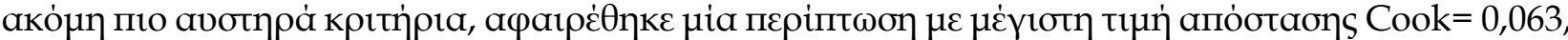

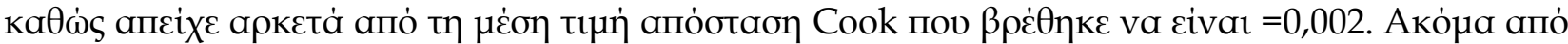

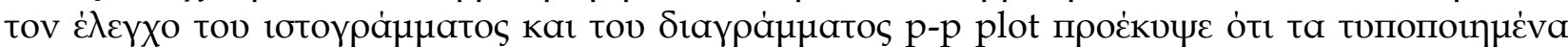

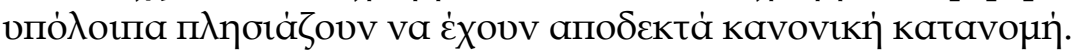

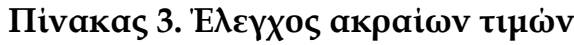

\begin{tabular}{|c|c|c|c|c|}
\hline 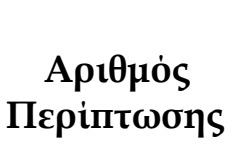 & 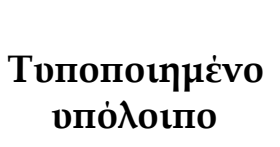 & 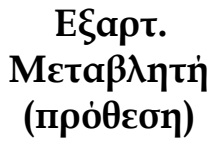 & 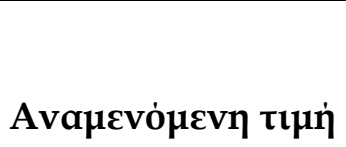 & Үпо̀ภочпо \\
\hline 120 & $-4,189$ & 1,67 & 5,1930 & $-3,52633$ \\
\hline 129 & $-3,569$ & 2,00 & 5,0047 & $-3,00475$ \\
\hline 29 & $-3,245$ & 1,67 & 4,3983 & $-2,73158$ \\
\hline 85 & $-3,084$ & 2,56 & 5,1519 & $-2,59637$ \\
\hline 68 & $-3,022$ & 2,78 & 5,3217 & $-2,54389$ \\
\hline 454 & 3,984 & 6,56 & 3,2017 & 3,35387 \\
\hline
\end{tabular}

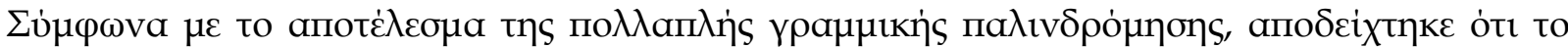

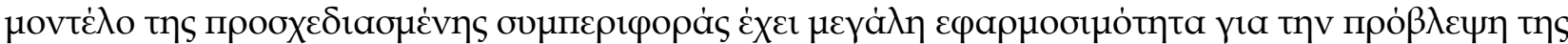

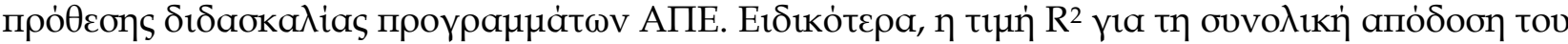

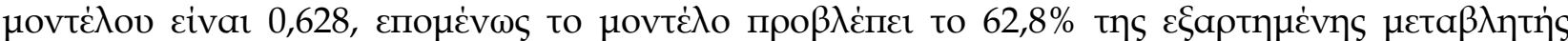

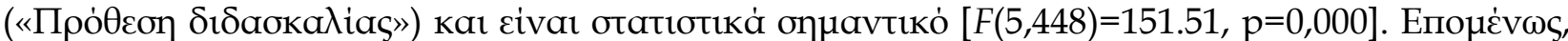

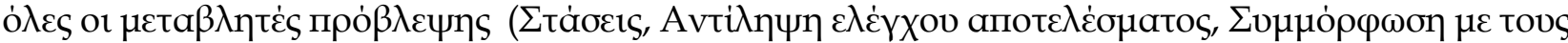

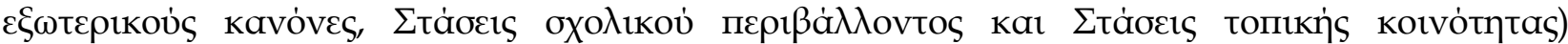

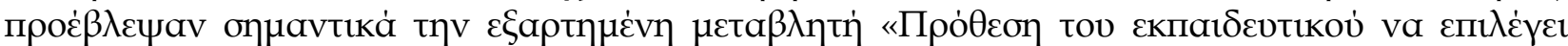
бХо

'Oбоv a

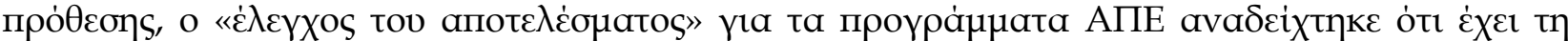

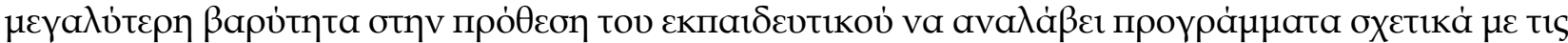

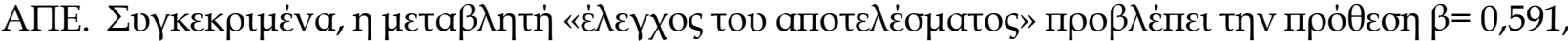

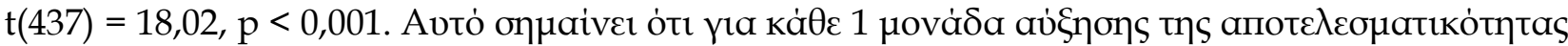

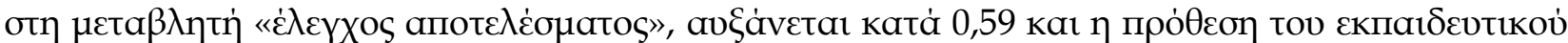

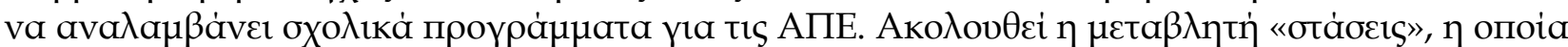




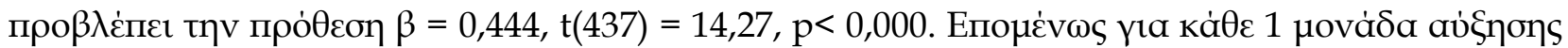

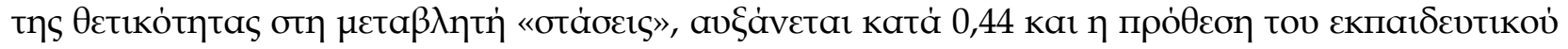

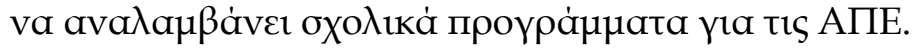

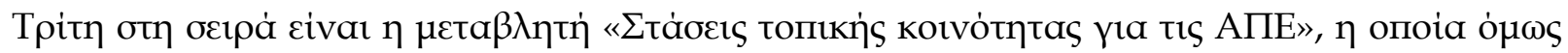

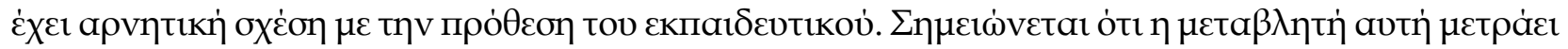

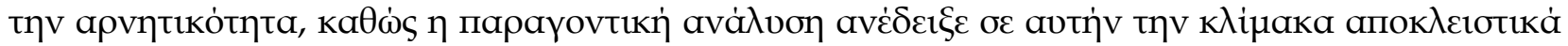

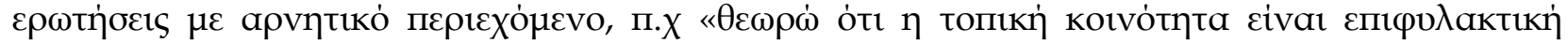

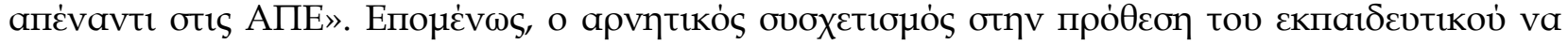

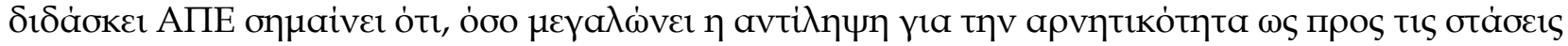

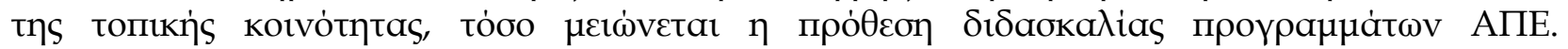

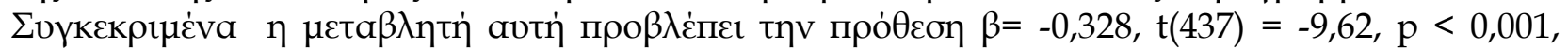

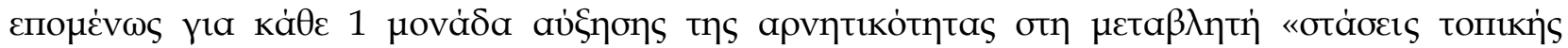

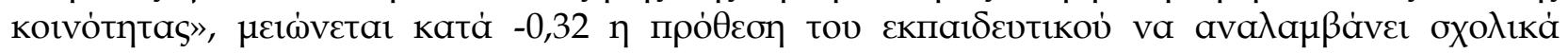

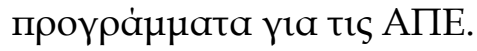

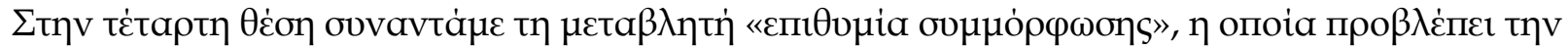

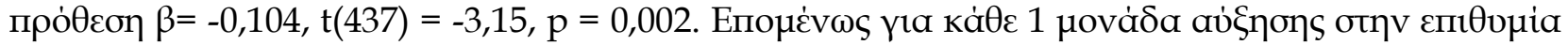

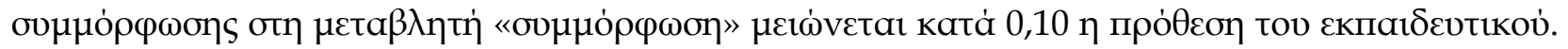

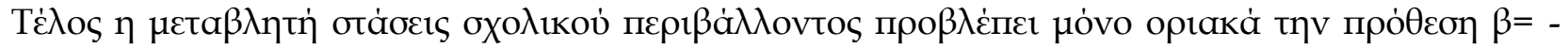
$0,089, \mathrm{t}(437)=-2,83, \mathrm{p}<0.005$ (Пivakas 4 ).

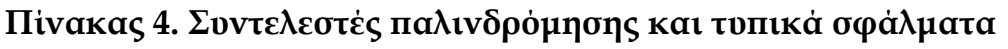

\begin{tabular}{|c|c|c|c|c|c|}
\hline \multirow[b]{2}{*}{ Model } & \multicolumn{2}{|c|}{$\begin{array}{c}\text { Unstandardized } \\
\text { Coefficients }\end{array}$} & \multicolumn{2}{|c|}{$\begin{array}{c}\text { Standardized } \\
\text { Coefficients }(\beta)\end{array}$} & \multirow[b]{2}{*}{ Sig. } \\
\hline & B & Std. Error & $\beta$ & $\mathrm{T}$ & \\
\hline$\overline{\Sigma \eta \mu \varepsilon i o ~ t o \mu \eta ̀ ่ ~(i n t e r c e p t) ~}$ & 1,719 & 0,315 & & 5,452 & ,000 \\
\hline 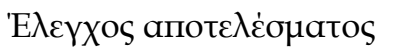 & 0,444 & 0,025 & 0,591 & 18,025 & ,000 \\
\hline ¿táocis & 0,549 & 0,038 & 0,444 & 14,272 & ,000 \\
\hline 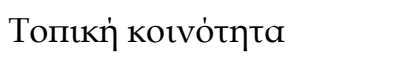 & $-0,301$ & 0,031 & $-0,328$ & $-9,627$ & ,000 \\
\hline$\Sigma v \mu \mu \dot{\rho} \rho \varphi \omega \sigma \eta$ & $-0,077$ & 0,024 & $-0,104$ & $-3,152$ &, 002 \\
\hline 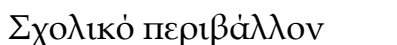 & $-0,104$ & 0,037 & $-0,089$ & $-2,833$ & ,005 \\
\hline
\end{tabular}

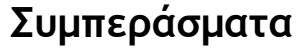

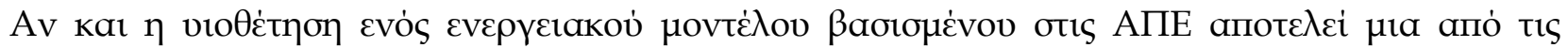

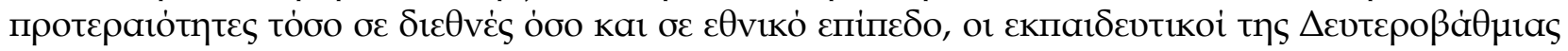

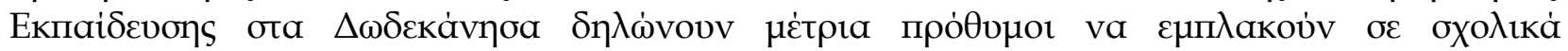

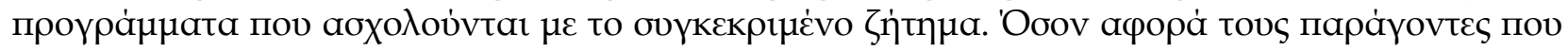

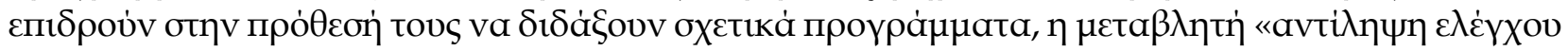

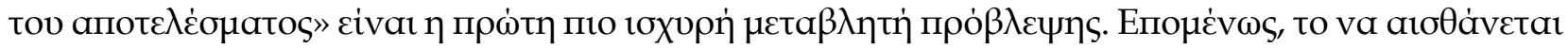

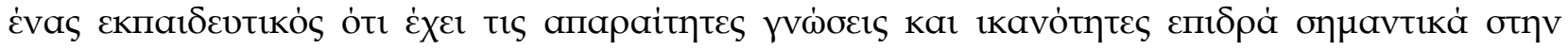

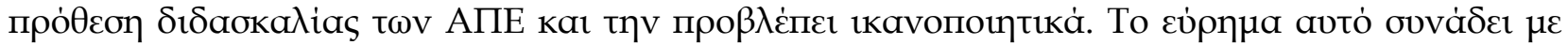

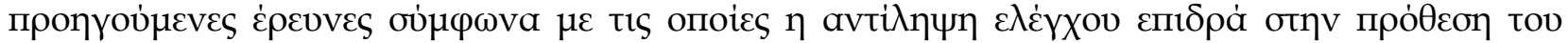

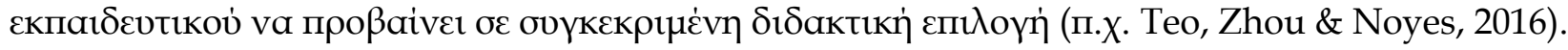

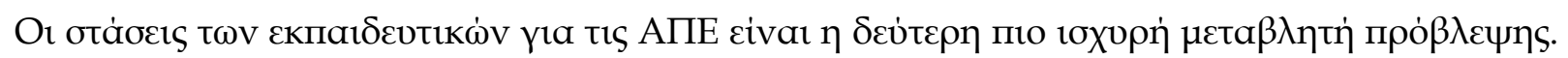

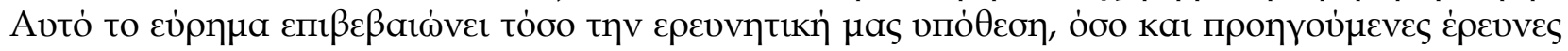

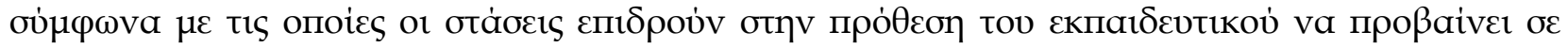

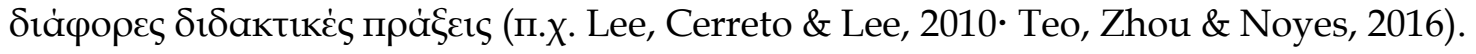

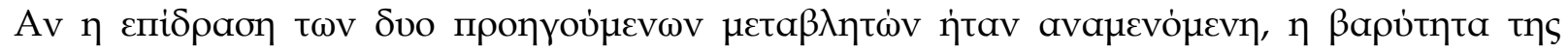

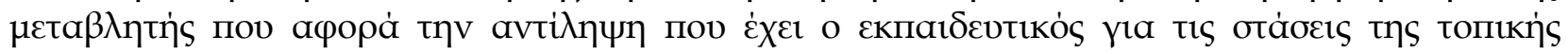

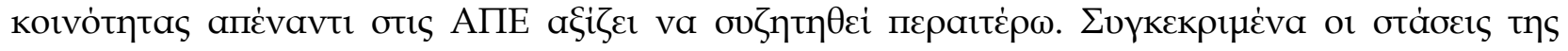

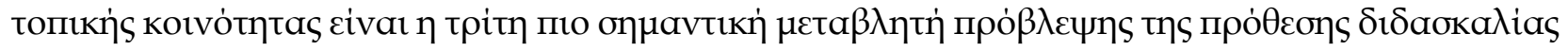




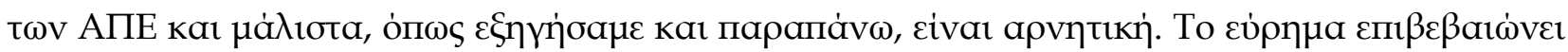

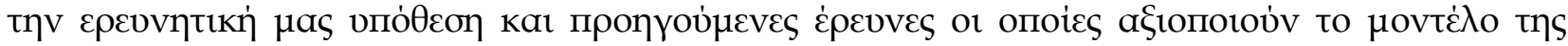

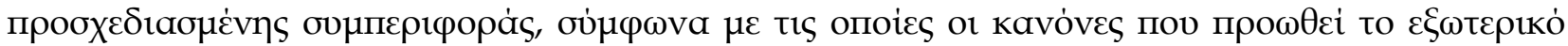

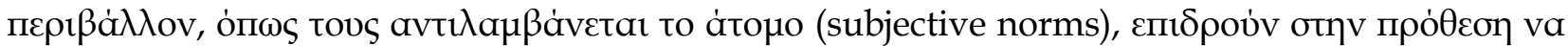

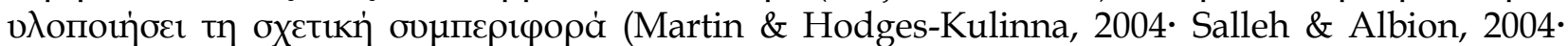
MacFarlane \& Woolfson, 2013· Salleh \& Laxman, 2015).

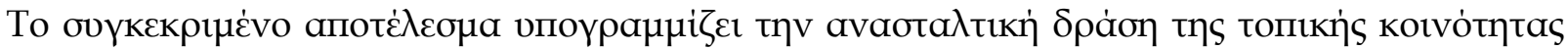

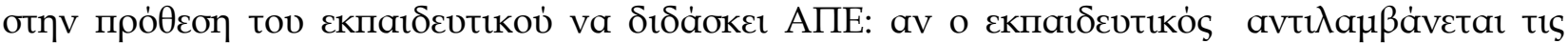

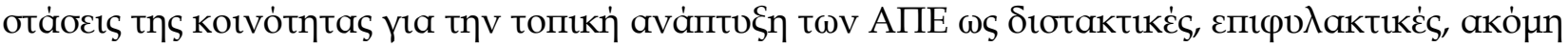

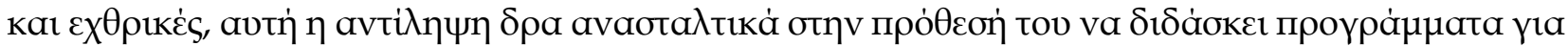
tıS АПЕ.

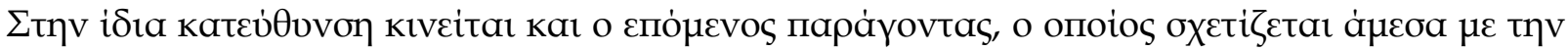

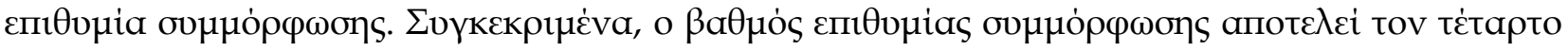

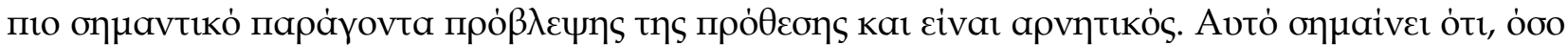

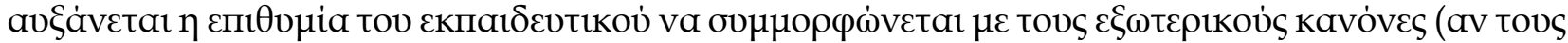

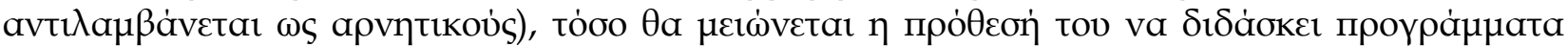

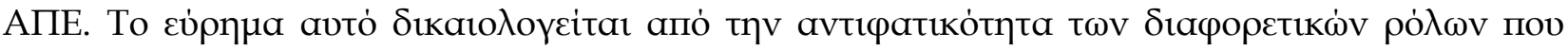

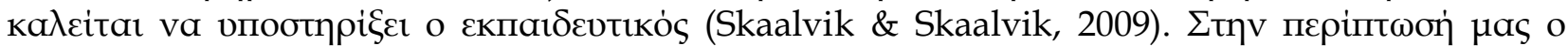

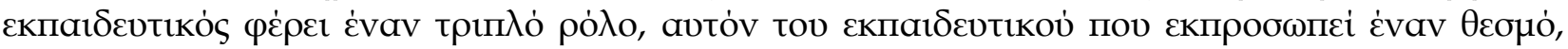

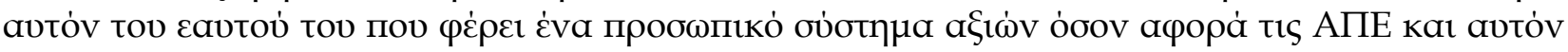

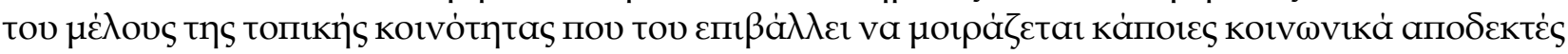

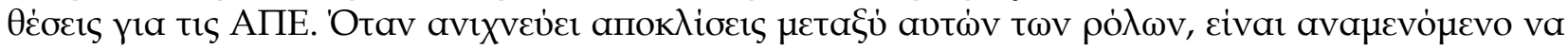

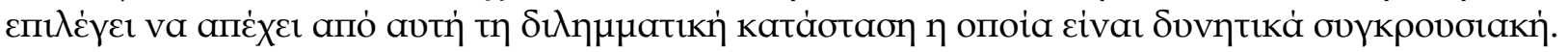

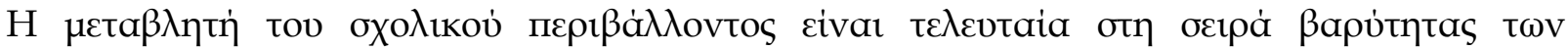

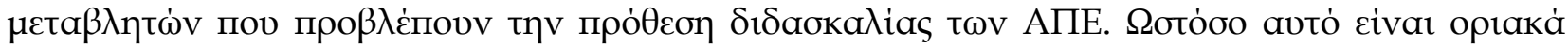

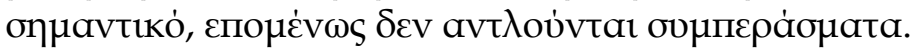

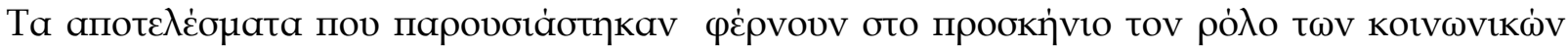

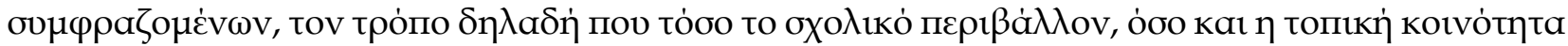

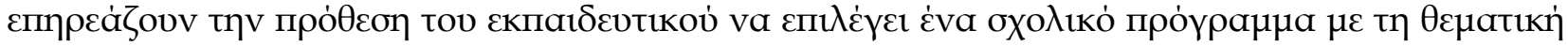

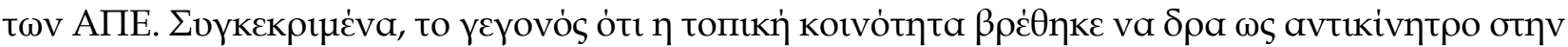

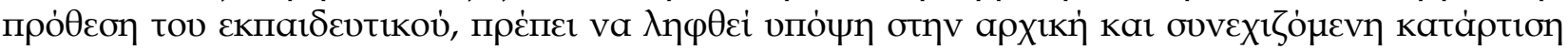

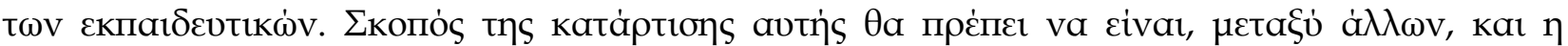

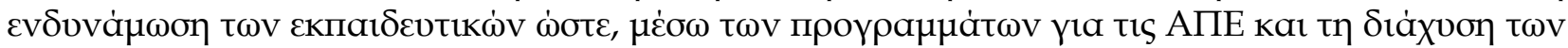

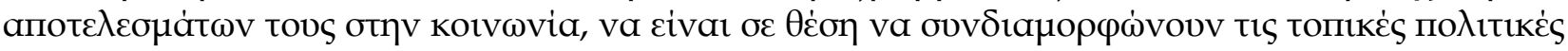

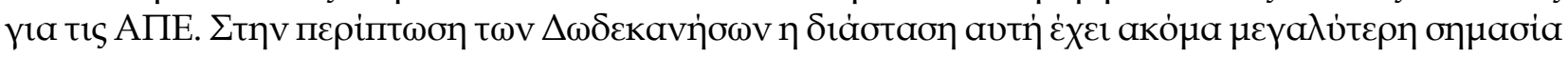

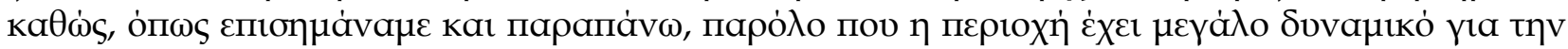

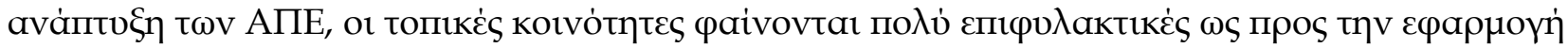

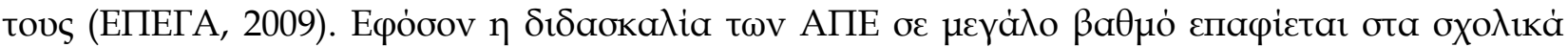

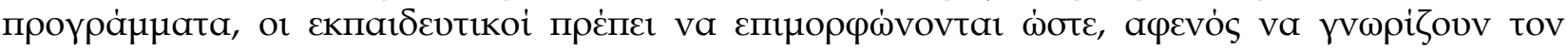

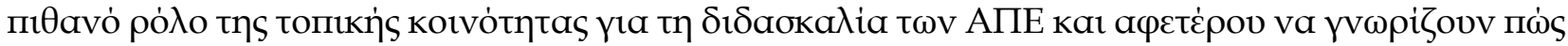

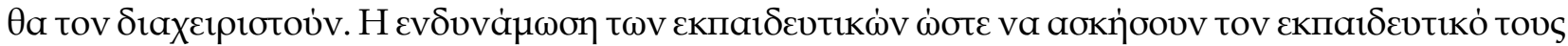

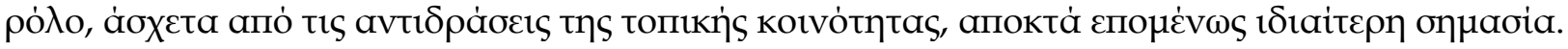

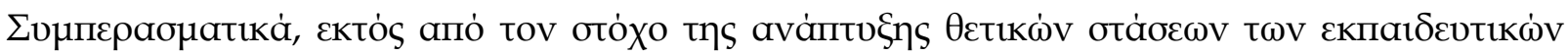

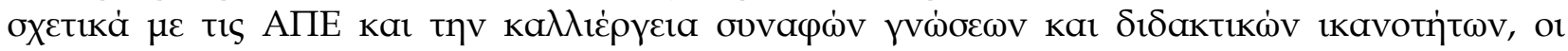

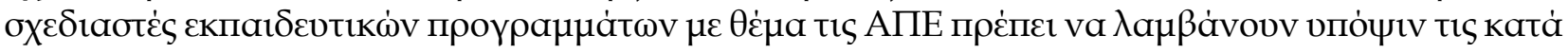

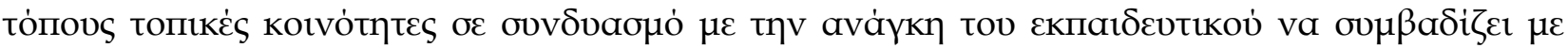

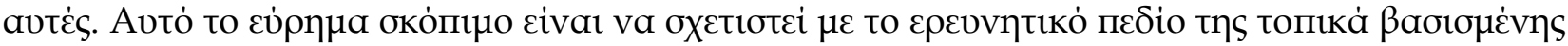

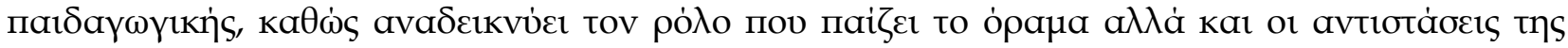

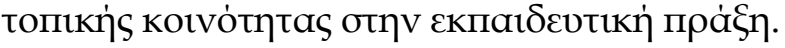




\section{SUMMARY IN ENGLISH}

The current study aims to investigate the factors that influence teachers' motivation to choose optional teaching topics related to Renewable Energy Sources (RES). 454 secondary education teachers from Dodecanese region, Greece, answered a questionnaire structured based on Planned Behavior Theory (Ajzen, 2002). Data were analyzed with multiple linear regression in order to investigate which factors influence teachers' motivation to teach RES related topics. A model was found to be statistically significant $(\mathrm{p}<.001)$, explaining $62.8 \%$ of the variance of teachers' motivation. Teachers' attitudes towards RES was found to be the strongest predictor of teachers' motivation, followed by perceived ability to teach RES topics and perceived attitudes of local community towards RES.

\section{Avạopźs}

Ajzen, I. (1991). The theory of planned behavior. Organizational Behavior and Human Decision Processes, 50, $179-211$.

Ajzen, I. (2002). Constructing a TpB Questionnaire: Conceptual and Methodological Considerations. (Revised January, 2006). Аvакті்

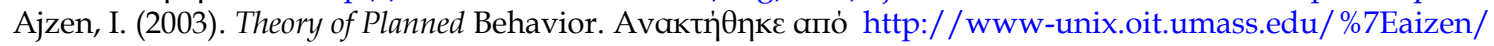

Alemayehu, B., Menkir, S., Basha, G. \& Nigatu, L. (2014). Students' and Teachers' Knowledge, Attitudes and Perceptions towards Bioenergy Resources. MIER Journal of Educational Studies, Trends \& Practices, 4(2), 183-194.

Balkar, B. (2015). The Relationships between organizational climate, innovative behavior and job performance of teachers. International Online Journal of Educational Sciences, 7(2), 81-92. https:/ / doi.org/10.15345/iojes.2015.02.007

Barry, J., Ellis, G. \& Robinson, C. (2008). Cool Rationalities and Hot Air: A Rhetorical Approach to Understanding Debates on Renewable Energy. Global Environmental Politics, 8(2), 67-98. https://doi.org/10.1162/glep.2008.8.2.67

Batel, S. \& Devine-Wright, P. (2015). Towards a better understanding of people's responses to renewable energy technologies: insights from social representations theory. Public Understanding of Science, 24(3), 311-325. https://doi.org/10.1177/0963662513514165

Burmeister, M. \& Eilks, I. (2013). Using participatory action research to develop a course module on Education for Sustainable Development in pre-service chemistry teacher education. CEPS Journal, 3(1), 59-78.

Cook, R. D. \& Weisberg, S. (1982). Residuals and influence in regression. New York: Chapman and Hall.

Cotton, M. \& Devine-Wright, P. (2010). NIMBYism and community consultation in electricity transmission network planning. In P. Devine-Wright (Ed.), Renewable energy and the public: From NIMBY to participation (pp. 115-130). London: Earthscan from Routledge.

Deci, E. L., Vallerand, R. J., Pelletier, L. G. \& Ryan, R. M. (1991). Motivation in education: The self-determination perspective. The Educational Psychologist, 26(3-4), 325-346. https://doi.org/10.1207/s15326985ep2603\&4_6

Devine-Wright, P. (2011). Place attachment and public acceptance of renewable energy: a tidal energy case study. Journal of Environmental Psychology, 31(4), 336-343. https:/ / doi.org/10.1016/j.jenvp.2011.07.001

Durbin, J. \& Watson, G.S. (1950). Testing for serial correlation in least squares regression, I. Biometrika, 37(3-4), 409-428. https://doi.org/10.1093/biomet/37.3-4.409

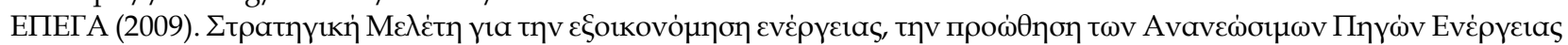

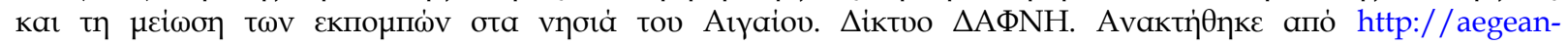
energy.gr/gr/ape/stratigiki-meleti.php

Fishbein, M. \& Ajzen, I. (1975). Belief, attitude, intention, and behavior. Reading, MA: Addison-Wesley.

Grace, M. \& Sharp, J. (2000). Exploring the actual and potential rhetoric-reality gaps in environmental education and their implications for pre-service teacher training. Environmental Education Research, 6(4), 331-345. https:// doi.org/10.1080/713664698

Gruenewald, D. A. (2016). Foundations of place: a multidisciplinary framework for place-conscious education. American Educational Research Journal, 40(3), 619-54. https:// doi.org/10.3102/00028312040003619

Guven, G., \& Sulun, Y. (2017). Pre-service teachers' knowledge and awareness about renewable energy. Renewable and Sustainable Energy Reviews, 80(C), 663-668. https:/ / doi.org/10.1016/j.rser.2017.05.286

Hasiloglou, A. M. (2017). The examining of prospective teachers views about renewable and non-renewable energy sources: A case study of Turkey. Educational Research and Reviews, 9(13), 411-416. https://doi.org/10.5897/ERR2013.1698

Hauser-Cram, P., Sirin, S. \& Stipek, D. (2003). When teachers' and parents' values differ: Teachers' ratings of academic competence in children from low-income families. Journal of Educational Psychology, 95(4), 813-820. https://doi.org/10.1037/0022-0663.95.4.813 
Lasky, S. (2005). A sociocultural approach to understanding teacher identity, agency and professional vulnerability in a context of secondary school reform. Teaching and Teacher Education, 21(8), 899-916. https://doi.org/10.1016/j.tate.2005.06.003\}

Lee, A. N., \& Nie, Y. (2017). Teachers' perceptions of school leaders' empowering behaviors and psychological empowerment: Evidence from a Singapore sample. Educational Management Administration E Leadership, $45(2), 260$ 283. https://doi.org/10.1177/1741143215578448

Lee, J., Cerreto, F. A. \& Lee, J. (2010). Theory of planned behavior and teachers' decisions regarding use of educational technology. Educational Technology \& Society, 13(1), 152-164. https://www.researchgate.net/publication/220374412

Leptokaridou, E.T., Vlachopoulos, S.P., \& Papaioannou, A. G. (2016). Experimental longitudinal test of the influence of autonomy-supportive teaching on motivation for participation in elementary school physical education. educational psychology, 36(7), 1135-1156. https://doi.org/10.1080/01443410.2014.950195

Li, H., Guo S., Cui, L., Yan, J., Liu, J. \& Wang, B. (2015). Review of renewable energy industry in Beijing: Development status, obstacles and proposals. Renewable and Sustainable Energy Reviews, 43, 711-725. https:// doi.org/10.1016/j.rser.2014.11.074\}

Liarakou, G., Gavrilakis, C. \& Flouri, E. (2009). Secondary school teachers' knowledge and attitudes towards renewable energy sources. Journal of Science Education and Technology, 18(2), 120-129. https://doi.org/10.1007/s10956-008-9137$\mathrm{z}$

MacFarlane, K.. \& Woolfson, L., M. (2013). Teacher attitudes and behavior toward the inclusion of children with social, emotional and behavioral difficulties in mainstream schools: An application of the theory of planned behavior. Teaching and Teacher Education, 29, 46-52. https://doi.org/10.1016/j.tate.2012.08.006

Martin J., \& Hodges-Kulinna, P. (2004). Self-Efficacy theory and the theory of planned behavior: teaching physically active physical education classes. Research Quarterly for Exercise and Sport, 75(3), 288-297. https://doi.org/ 10.1080/02701367.2004.10609161\}

Moula M. E., Maula J., Hamdy, M., Fang, T., Jung, N. \& Lahdelma, R. (2013). Researching social acceptability of renewable energy technologies in Finland. International Journal of Sustainable Built Environment, 2(1), 89-98. https:// doi.org/10.1016/j.ijsbe.2013.10.001

O'Brennan, L. M., Bradshaw, C. P. \& Furlong, M. J. (2014). Influence of classroom and school climate on teacher perceptions of student problem behavior. School Mental Health, 6(2) 125-136. https://doi.org/10.1007/s12310-0149118-8

Oikonomou, E. K., Kilias, V., Goumas, A., Rigopoulos, A., Karakatsani, E., Damasiotis, M., Papastefanakis, D. \& Marini, N. (2009). Renewable energy sources (RES) projects and their barriers on a regional scale: The case study of wind parks in the Dodecanese islands, Greece. Energy Policy, 37(11), 4874-4883.

Rubie-Davies, C. M., Flint, A. \& McDonald, L. (2012). Teacher beliefs, teacher characteristics and school contextual factors: What are the relationships? British Journal of Educational Psychology, 82(2), 270 - 288. https://doi.org/ 10.1111/j.2044-8279.2011.02025.x

Sadik, F. \& Sadik, S. (2014). A study on environmental knowledge and attitudes of teacher candidates. Social and Behavioral Sciences, 116(21), 2379-2385. https:/ / doi.org/10.1016/j.sbspro.2014.01.577

Salleh, S. \& Albion, P. (2004). Using the theory of planned behavior to predict Bruneian teachers' intentions to use ICT in teaching. In proceedings of the 15th International Conference of the Society for Information Technology E Teacher Education (SITE 2004), 1-6 Mar, Atlanta, Georgia, USA (pp. 1389-1396). Association for the Advancement of Computing in Education (AACE)

Salleh, S. \& Laxman, K. (2015). Examining the effect of external factors and context-dependent beliefs of teachers in the use of ict in teaching: using an elaborated theory of planned behavior. Journal of Educational Technology Systems, 43(3) 289-319. https://doi.org/10.1177/0047239515570578

Seraphin, K. D., Philippoff, J. \& Parisky, A., Degnan K. \& Papini Warren, D. (2013). Teaching energy science as inquiry: reflections on professional development as a tool to build inquiry teaching skills for middle and high school teachers. Journal of Science Education and Technology, 22(3), 235-251. https://doi.org/10.1007/s10956-012-9389-5

Sharifah-Norhaidah, S. (2006). Exploring environmental behaviours, attitudes and knowledge among university students: positioning the concept of sustainable development within Malaysian education. Journal of Science and Mathematics Education in Southeast Asia, 29(1), 79-97.

Skaalvik, E. M. \& Skaalvik, S. (2009). Does school context matter? Relations with teacher burnout and job satisfaction. Teaching and Teacher Education. An International Journal of Research and Studies, 25(3), 518-524. https://doi.org/10.1016/j.tate.2008.12.006

Skaalvik, E. M. \& Skaalvik, S. (2011). Teacher job satisfaction and motivation to leave the teaching profession: Relations with school context, feeling of belonging, and emotional exhaustion. Teaching and Teacher Education: An International Journal of Research and Studies, 27(6), 1029-1038. https://doi.org/10.1016/j.tate.2011.04.001

Skelton, A. (2012). Value conflicts in higher education teaching. Teaching in higher education, 17(3), $257-268$. https://doi.org/10.1080/13562517.2011.611875

Spiropoulou, D., Antonakaki, T., Kontaxaki, S. \& Bouras, S. (2007). Primary teachers' literacy and attitudes on education for sustainable development. Journal of Science Education and Technology, 16(5), 443-450. https:/ / doi.org/10.1007/s10956-007-9061-7

Stanec, A. (2009). The theory of planned behavior: predicting teachers' intentions and behavior during fitness testing. Journal of Teaching Physical Education, 28(3), 255-271. https:// doi.org/10.1123/jtpe.28.3.255 
Sun, L., Yung, E. H. K., Chan, E. H. W. \& Zhu, D. (2016). Issues of NIMBY conflict management from the perspective of stakeholders: A case study in Shanghai. Habitat International, 53, $133-141$. https://doi.org/10.1016/j.habitatint.2015.11.013

Teo, T., Zhou, M. \& Noyes, J. (2016). Teachers and technology: development of an extended theory of planned behavior. Educational Technology Research and Development, 64(6), 1033-1052. https://doi.org/10.1007/s11423-016-9446-5

Tortop, H. S. (2013). Teaching techniques and activities for the education of the gifted young scientist: meaningful field trip in education of the renewable energy technologies. Journal for the Education of the Young Scientist and Giftedness, 1(1), 8-15. https:/ / doi.org/10.17478/JEYSG.201318998

Tsigilis, N., Tsioumis, K. \& Gregoriadis, A. (2006). Prospective early childhood educators' attitudes toward teaching multicultural classes: a planned behavior theory perspective. Journal of Early Childhood Teacher Education, 27(3), 265273. https://doi.org/10.1080/10901020600843624

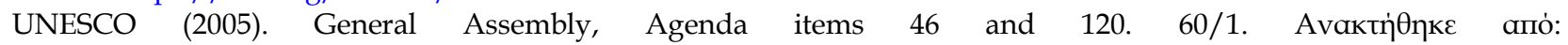
https://www.un.org/en/development/desa/population/migration/generalassembly/docs/globalcompact/A_R ES_60_1.pdf

Woodhouse, J. \& Knapp, C. E. (2013). Place-Based Curriculum and Instruction. ERIC Clearinghouse on Rural Education

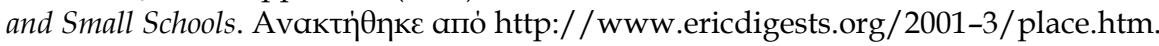

Zyadin, A., Puhakka, A., Ahponen, P. \& Pelkonen, P. (2014). Secondary school teachers' knowledge, perceptions, and attitudes toward renewable energy in Jordan. Renewable Energy, 62(C), 341-348. https://doi.org/ 10.1016/j.renene.2013.07.033\}

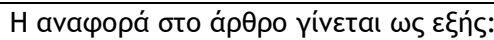

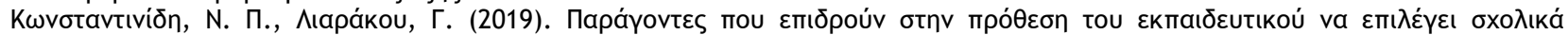

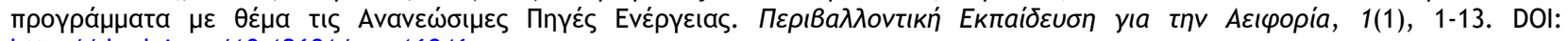
http://dx.doi.org/10.12681/ees.16846

https://ejournals.epublishing.ekt.gr/index.php/enveducation/index 